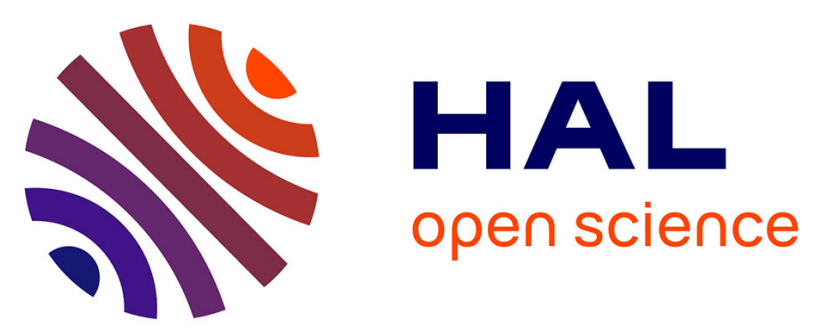

\title{
Investigating the oxygenation of brain arteriovenous malformations using quantitative susceptibility mapping
}

Emma Biondetti, Alvaro Rojas-Villabona, Magdalena Sokolska, Francesca B

Pizzini, Hans Rolf Jäger, David L Thomas, Karin Shmueli

\section{- To cite this version:}

Emma Biondetti, Alvaro Rojas-Villabona, Magdalena Sokolska, Francesca B Pizzini, Hans Rolf Jäger, et al.. Investigating the oxygenation of brain arteriovenous malformations using quantitative susceptibility mapping. NeuroImage, 2019, 199, pp.440-453. 10.1016/j.neuroimage.2019.05.014 . hal02284294

\author{
HAL Id: hal-02284294 \\ https: / hal.sorbonne-universite.fr/hal-02284294
}

Submitted on 11 Sep 2019

HAL is a multi-disciplinary open access archive for the deposit and dissemination of scientific research documents, whether they are published or not. The documents may come from teaching and research institutions in France or abroad, or from public or private research centers.
L'archive ouverte pluridisciplinaire HAL, est destinée au dépôt et à la diffusion de documents scientifiques de niveau recherche, publiés ou non, émanant des établissements d'enseignement et de recherche français ou étrangers, des laboratoires publics ou privés. 


\section{Investigating the Oxygenation of Brain Arteriovenous Malformations Using Quantitative Susceptibility Mapping}

\section{Author names and affiliations}

Emma Biondetti ${ }^{\mathrm{a}, 1}$, Alvaro Rojas-Villabona ${ }^{\mathrm{b}, \mathrm{c}, 2}{ }^{2}$, Magdalena Sokolska ${ }^{\mathrm{d}}$, Francesca B. Pizzini ${ }^{\mathrm{e}}$, Hans Rolf Jäger ${ }^{\mathrm{f}, \mathrm{g}}$, David L. Thomas ${ }^{\mathrm{g}, \mathrm{h}}$, and Karin Shmueli ${ }^{\mathrm{a}}$

aMRI Group, Department of Medical Physics and Biomedical Engineering, Malet Place Engineering Building, University College London, WC1E 6BT, London, UK

${ }^{\text {b}}$ The Gamma Knife Centre at Queen Square, National Hospital for Neurology and Neurosurgery, Queen Square, WC1N 3BG, London, UK

'Department of Neurosurgery, National Hospital for Neurology and Neurosurgery, Queen Square WC1N 3BG, London, UK

${ }^{\mathrm{d} M e d i c a l}$ Physics and Biomedical Engineering, University College London Hospitals, NW1 2BU, London, UK

eNeuroradiology Unit, Department of Diagnostics and Pathology, Verona University Hospital, Piazzale Stefani, 37126, Verona, Italy

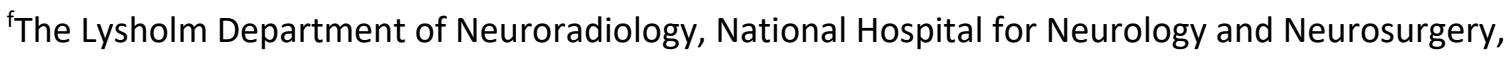
Queen Square, WC1N 3BG, London, UK

${ }^{g}$ Neuroradiological Academic Unit, UCL Institute of Neurology, University College London, Queen Square, WC1N 3BG, London, UK

hLeonard Wolfson Experimental Neurology Centre, UCL Institute of Neurology, University College London, Queen Square, WC1N 3BG, London, UK

\section{Corresponding author}

Emma Biondetti

Postal address: Institut du Cerveau et de la Moelle épinière (ICM), Centre de Neurolmagerie de Recherche (CENIR), Hôpital Pitié-Salpêtrière, 47 Boulevard de l'Hôpital, 75013 Paris, France

E-mail: emma.biondetti@icm-institute.org

\footnotetext{
${ }^{1}$ Present address: Institut du Cerveau et de la Moelle épinière (ICM), Centre de Neurolmagerie de Recherche (CENIR), Sorbonne Universités, CNRS UMR 7225, INSERM U 1127, Hôpital Pitié-Salpêtrière, 47 Boulevard de I'Hôpital, 75013, Paris, France

2 Present address: Neurosurgery Department, New Victoria Wing, Royal Victoria Infirmary, Queen Victoria Road, NE1 4LP, Newcastle upon Tyne, UK
} 


\section{Abstract}

Brain arteriovenous malformations (AVMs) are congenital vascular anomalies characterized by arteriovenous shunting through a network of coiled and tortuous vessels. Because of this anatomy, the venous drainage of an AVM is hypothesized to contain more oxygenated, arterialized blood than healthy veins. By exploiting the paramagnetic properties of deoxygenated hemoglobin in venous blood using magnetic resonance imaging (MRI) quantitative susceptibility mapping (QSM), we aimed to explore venous density and oxygen saturation $\left(\mathrm{SvO}_{2}\right)$ in patients with a brain AVM.

We considered three groups of subjects: patients with a brain AVM before treatment using gamma knife radiosurgery (GKR); patients three or more years post-GKR treatment; and healthy volunteers. First, we investigated the appearance of AVMs on QSM images. Then, we investigated whether QSM could detect increased $\mathrm{SvO}_{2}$ in the veins draining the malformations.

In patients before $\mathrm{GKR}$, venous density, but not $\mathrm{SvO}_{2}$, was significantly larger in the hemisphere containing the AVM compared to the contralateral hemisphere $(p=0.03)$. Such asymmetry was not observed in patients after GKR or in healthy volunteers. Moreover, in all patients before GKR, the vein immediately draining the AVM nidus had a higher $\mathrm{SVO}_{2}$ than healthy veins. Therefore, QSM can be used to detect $\mathrm{SvO}_{2}$ alterations in brain AVMs. However, since factors such as flow-induced signal dephasing or the presence of hemosiderin deposits also strongly affect QSM image contrast, AVM vein segmentation must be performed based on alternative MRI acquisitions, e.g., time of flight magnetic resonance angiography or $\mathrm{T}_{1}$-weighted images.

This is the first study to show, non-invasively, that AVM draining veins have a significantly larger $\mathrm{SvO}_{2}$ than healthy veins, which is a finding congruent with arteriovenous shunting.

\section{Keywords}

Magnetic resonance imaging, quantitative susceptibility mapping, arteriovenous malformation, venous oxygen saturation, venous density 


\section{Introduction}

Arteriovenous malformations (AVMs) are high-flow congenital vascular anomalies characterized by arteriovenous shunting with the lack of an intervening capillary bed. In an AVM, arteries and veins are directly connected through a network of coiled and tortuous vessels called a nidus (Friedlander, 2007). In a healthy vascular network, the largest blood pressure drops occur in the capillary bed (Gould et al., 2017) whose hemodynamics and tortuous spatial organization enable a relatively uniform exchange of oxygen and nutrients between blood and the surrounding tissue. Therefore, in an AVM, the lack of capillaries causes the presence of mixed arterial and venous blood in the draining veins (Katayama et al., 1994) with higher velocity and often also higher pressure compared to healthy veins. The increased blood pressure, in particular, is a risk for vessel ruptures and hemorrhages (Friedlander, 2007).

Selected cases of brain AVMs can be treated non-invasively using gamma knife radiosurgery (GKR), which uses a high dose of highly-focused gamma radiation to induce AVM obliteration with minimal damage to the surrounding tissue (Starke et al., 2017). On average, AVM obliteration occurs over a period of three years with reported success rates of 65\% (Kano et al., 2012; Koltz et al., 2013; Starke et al., 2017).

Digital subtraction angiography (DSA) is the gold standard imaging method for delineating the radiosurgical target before treatment with GKR (Pollock et al., 2011; Starke et al., 2017) and verifying the degree of success of GKR (Pollock et al., 2011). Magnetic resonance imaging (MRI) acquisitions have been used to gain additional information on the anatomy and location of brain AVMs compared to DSA. For example, it has been shown that the joint use of time-of-flight (TOF) magnetic resonance angiography (MRA) and DSA increases the accuracy of AVM nidus delineation (Bednarz et al., 2000; Rojas-Villabona et al., 2017).

A further advantage of MRI over DSA is its potential to provide information on AVM pathophysiology. Because of their anatomy, the veins draining an AVM are expected to have a higher venous oxygen saturation $\left(\mathrm{SvO}_{2}\right)$, which has been indirectly evidenced by the finding of "red veins" intraoperatively and by "downstream" jugular bulb oxygenation measurements (Katayama et al., 1994). Further indirect evidence suggesting altered oxygen exchange between blood flowing through an AVM and the surrounding tissue has been obtained using MRI arterial spin labeling (ASL) (Alsop et al., 2015). It has been shown (Kukuk et al., 2010; Le et al., 2012; Wolf et al., 2008) that the veins draining an AVM often appear unusually bright on an ASL image due to their residual unexchanged water content. However, the oxygenation of blood in veins immediately draining an

Abbreviations: AP, anterior-posterior; ASL, arterial spin labelling; AVM, arteriovenous malformation; DSA, digital subtraction angiography; DV, draining vein; FA, flip angle; FLIRT, FMRIB's Linear Image Registration Tool; FSL, Oxford Centre for Functional MRI in the Brain Software Library; FoV, field of view; gad, gadolinium; GKR, gamma knife radiosurgery; GRE, gradient-recalled echo; ICBM, International Consortium for Brain Mapping; MIP, maximum intensity projection; MNI, Montréal Neurological Institute; $\mathrm{MRI}$, magnetic resonance imaging; MRA, magnetic resonance angiography; MVF, multiscale vessel filtering; OEF, oxygen extraction fraction; PCA, phase contrast angiography; PET, positron emission tomography; PLIC, posterior limb of the internal capsule; REST, regional saturation technique; ROI, region of interest; RL, right-left; SD, standard deviation; SEM, standard error of the mean; SENSE, sensitivity encoding; SI, superior-inferior; SSS, superior sagittal sinus; SWI, susceptibility-weighted imaging; TE, echo time; TOF, time-of-flight; TR, repetition time; QSM, quantitative susceptibility mapping. 
AVM has never been directly or non-invasively measured. The magnetic susceptibility $(\chi)$ of paramagnetic (i.e., $\chi>0$ ) deoxyhemoglobin in venous blood is proportional to $\mathrm{SvO}_{2}$ (Fan et al., 2014; Jain et al., 2012; Spees et al., 2001; Weisskoff and Kiihne, 1992; Xu et al., 2014). Therefore, this study aimed to measure the $\mathrm{SvO}_{2}$ in veins directly draining brain AVMs non-invasively using quantitative susceptibility mapping (QSM) (Liu et al., 2009; Shmueli et al., 2009; Wang and Liu, 2015; Wharton and Bowtell, 2010). Moreover, by designing the gradient-recalled echo (GRE) protocol for QSM with a short first echo time (TE), this study aimed to assess whether the shortest TE magnitude image enabled visualization of in-flow effects in the arteries feeding the AVM nidus. If so, GRE protocols for QSM of vascular malformations could allow simultaneous acquisition of complementary arterial and venous contrasts.

To enable MRI QSM measurement of $\mathrm{SvO}_{2}$ in an AVM's draining veins, identifying these veins on a $\chi$ map is essential. The arteries feeding an AVM would not be expected to appear on a QSM image as arteries contain diamagnetic (i.e., $\chi<0$ ) fully oxygenated oxyhemoglobin, which does not cause a detectable $\chi$ shift compared to the diamagnetic brain parenchyma (Duyn and Schenck, 2017; Jain et al., 2012). Similarly, because of their mixed content of arterial and venous blood, the veins draining an AVM would be expected to be less visible on a $\chi$ map than healthy veins.

The possibility of identifying brain AVMs and other vascular malformations on $\chi$-based images has been demonstrated using susceptibility-weighted imaging (SWI) (Haacke et al., 2004). In previous studies, SWI helped delineate brain AVMs (Essig et al., 1999; Gasparotti et al., 2011; George et al., 2010; Hodel et al., 2013; Huisman et al., 2010; Jagadeesan et al., 2011; Liu et al., 2015; Nabavizadeh et al., 2014; Reichenbach et al., 1998; Reichenbach and Haacke, 2001), arteriovenous fistulas (Gasparotti et al., 2011; Hodel et al., 2013; Jagadeesan et al., 2011; Nabavizadeh et al., 2014), capillary telangiectasias (Gelal et al., 2014; Lee et al., 1999; Tong et al., 2008), cavernous malformations (Dammann et al., 2013; Huisman et al., 2010; Knerlich-Lukoschus et al., 2015; Lee et al., 1999; Reichenbach et al., 2001; Thomas et al., 2008; Tong et al., 2008) and developmental venous anomalies (Fushimi et al., 2008; Horsch et al., 2014; Huisman et al., 2010; Liu et al., 2015; Reichenbach and Haacke, 2001; Thomas et al., 2008). A limitation of SWI is that it is purely a qualitative method because of the non-local nature and tissue geometry/orientation dependence of the GRE signal phase. Moreover, veins sometimes appear artifactually doubled on SWI, because of the dipole-like phase distributions around high- $\chi$ regions (Dymerska et al., 2018; Nissi et al., 2015). By calculating the underlying $\chi$ distribution, QSM overcomes the non-locality and orientationdependence of tissue phase and SWI (Shmueli et al., 2009). Finally, a previous study using QSM (Liu et al., 2015) detected the presence of the abnormal vasculature of a brain AVM in one patient, but did not attempt to delineate single vessels draining the malformation or measure $\mathrm{SvO}_{2}$.

In this study, we characterized the appearance of AVMs on susceptibility maps and measured $\chi$ based $\mathrm{SVO}_{2}$ in the AVM draining veins in two groups of patients before and after treatment with GKR. To enable comparison with normal appearing venous vasculature, we also compared the susceptibility maps and $\mathrm{SvO}_{2}$ measurements obtained in pre-GKR and post-GKR patients with those from healthy volunteers. 


\begin{tabular}{|c|c|c|c|c|c|}
\hline Parameter & $\begin{array}{l}\text { Sequence: } \\
\text { Units }\end{array}$ & (a) 2D GRE & (b) 3D GRE & $\begin{array}{c}\text { (c) Gad-TOF } \\
\text { MRA }\end{array}$ & $\begin{array}{l}\text { (d) } \text { Gad-T }_{1-} \\
\text { weighted }\end{array}$ \\
\hline $\begin{array}{l}\text { Pulse sequence } \\
\text { type }\end{array}$ & & $\overline{G R E}$ & GRE & GRE & $\overline{G R E}$ \\
\hline Imaging type & & $2 \mathrm{D}$ & $3 D$ & $3 D$ & $3 D$ \\
\hline $\mathrm{TE}(\mathrm{s})$ & $\mathrm{ms}$ & $\begin{array}{c}7.1,13.4,19.7 \\
26,32.3,38.6 \\
44.9,51.2 \\
57.6,63.9\end{array}$ & $\begin{array}{c}3.43,9.76,16 \\
22.4,28.7,35 \\
41.4\end{array}$ & 3.45 & 2.12 \\
\hline TR & $\mathrm{ms}$ & 5635 & 46 & 25 & 25 \\
\hline FA & deg & 90 & 17 & 20 or 25 & 30 \\
\hline Acquisition time & $\min : s$ & $08: 24$ & $08: 37$ & $08: 54$ & $18: 28$ \\
\hline Pixel Bandwidth & $\mathrm{Hz} /$ pixel & 217 & 210 & 216 & 157 \\
\hline FoV & $\mathrm{mm}^{3}$ & $240 \times 180 \times 144$ & $246 \times 184 \times 144$ & $250 \times 250 \times 50$ & $210 \times 210 \times 150$ \\
\hline $\begin{array}{l}\text { Acquired voxel } \\
\text { size }\end{array}$ & $\mathrm{mm}^{3}$ & $1 \times 1 \times 2$ & $1.1 \times 1.1 \times 1.1$ & $0.3 \times 0.44 \times 1$ & $0.82 \times 0.82 \times 1.5$ \\
\hline $\begin{array}{l}\text { Reconstructed } \\
\text { voxel size }\end{array}$ & $\mathrm{mm}^{3}$ & $1 \times 1 \times 2$ & $1.1 \times 1.1 \times 1.1$ & $0.13 \times 0.13 \times 0.5$ & $0.82 \times 0.82 \times 1.5$ \\
\hline $\begin{array}{l}\text { Acquisition } \\
\text { matrix }\end{array}$ & $\mathrm{mm}^{3}$ & $240 \times 180 \times 72$ & $224 \times 167 \times 131$ & $832 \times 568 \times 50$ & $256 \times 256 \times 100$ \\
\hline $\begin{array}{l}\text { Reconstruction } \\
\text { matrix }\end{array}$ & $\mathrm{mm}^{3}$ & $240 \times 240 \times 72$ & $224 \times 224 \times 131$ & $\begin{array}{c}1936 \times 1936 \times \\
100 \\
\end{array}$ & $256 \times 256 \times 100$ \\
\hline Slice orientation & - & Transverse & Transverse & Transverse & Transverse \\
\hline $\begin{array}{l}\text { Phase encoding } \\
\text { direction }\end{array}$ & - & $\mathrm{RL}$ & $\mathrm{RL}$ & $\mathrm{RL}$ & $\mathrm{RL}$ \\
\hline $\begin{array}{l}\text { Slice select (2D) } \\
\text { / partition } \\
\text { encoding } \\
\text { direction (3D) }\end{array}$ & - & $\mathrm{SI}$ & $\mathrm{SI}$ & $\mathrm{SI}$ & SI \\
\hline $\begin{array}{l}\text { Parallel imaging } \\
\text { method }\end{array}$ & - & $\begin{array}{l}\text { SENSE in the RL } \\
\text { direction, } \\
\text { factor }=2\end{array}$ & $\begin{array}{l}\text { SENSE in the RL } \\
\text { direction, } \\
\text { factor }=2\end{array}$ & $\begin{array}{l}\text { SENSE in the RL } \\
\text { direction, factor } \\
=2\end{array}$ & None \\
\hline Fat suppression & - & No & No & No & No \\
\hline Brain coverage & - & Whole brain & Whole brain & $\begin{array}{c}\text { Restricted to the } \\
\text { AVM }\end{array}$ & Whole brain \\
\hline $\begin{array}{l}\text { Flow } \\
\text { compensation }\end{array}$ & - & $\begin{array}{c}\text { First TE, AP } \\
\text { direction }\end{array}$ & - & - & - \\
\hline Slab(s) & & 1 & 1 & 4 & 1 \\
\hline REST slab & - & Yes & No & & \\
\hline $\begin{array}{l}\text { Post-contrast } \\
\text { (gadolinium) }\end{array}$ & - & No & No & Yes & Yes \\
\hline
\end{tabular}

\section{Materials and methods}

Where not otherwise stated, the calculations and analyses were conducted in Matlab (R2017b, The MathWorks, Natick, MA, USA).

\subsection{Data and Code Availability Statement}

In compliance with the requirements of the funding bodies and the institutional ethics approval policies, the data and code that support the findings of this study are available from the corresponding author upon reasonable request. 


\subsection{Image Acquisition}

For this study, three groups of subjects were scanned: fifteen with a brain AVM before treatment with GKR (pre-GKR group, IDs: AVM01-15, average age/age range: 38/18-62 years, ten females), seventeen with a brain AVM treated using GKR at least three years before the scan (post-GKR group, IDs: GKR01-17, average age/age range: 37/16-70 years, nine females) and thirteen healthy volunteers (IDs: HV01-13, average age/age range: 33/22-54 years, four females).

Three patients in the pre-GKR group had undergone partial glue embolization before the MRI examination, one of them on two occasions, and none of them had undergone surgical AVM excision. In the post-GKR group, there was a complete obliteration of the AVM after GKR except in four patients (GKR02, GKR04, GKR08 and GKR12).

The patients in the pre-GKR group and the healthy volunteers were scanned on a Philips Achieva 3T system at the Queen Square Multiple Sclerosis Centre (UCL Institute of Neurology, London, UK) using a 32-channel radiofrequency head coil. The patients in the post-GKR group were scanned on a Philips Achieva 3T system at Verona University Hospital (Neuroradiology Unit, Department of Diagnostic and Pathology, Verona University Hospital, Verona, Italy) using an 8-channel radiofrequency head coil.

The local ethics review boards approved all the scanning sessions and all subjects provided written informed consent. All the pre-GKR patients were scanned using a 3D multi-echo GRE protocol (Table 1b) optimized for QSM of brain AVMs. Six post-GKR patients were also scanned using the same 3D protocol, whereas eleven post-GKR patients were scanned using a 2D multi-echo GRE protocol (Table 1a), which had been previously developed for $T_{2}^{*}$ mapping. Of the thirteen healthy volunteers, ten were scanned using both the 2D and 3D GRE protocols, one with only the 3D protocol and two with only the 2D protocol.

In this study, the images of two healthy volunteers (HV1-2) and two post-GKR patients (GKRO4 and GKR09) were erroneously acquired with the transverse plane oblique (i.e., not perpendicular) to $\mathbf{B}_{0}$. Here, the image orientation was rotated to obtain alignment of the transverse imaging plane with the anterior-posterior commissure line. For these cases, the rotations needed to be accounted for in the QSM processing pipeline.

In addition to the GRE protocols, both the pre-GKR and post-GKR patients were scanned using a gadolinium-enhanced TOF MRA protocol (gad-TOF MRA, see Table 1c for acquisition details). Additionally, the pre-GKR patients were scanned using a post-gadolinium $\mathrm{T}_{1}$-weighted protocol (gad$\mathrm{T}_{1}$-weighted, Table $1 \mathrm{~d}$ ). These two additional scans were designed as a part of a comprehensive protocol for MRI-based radiosurgical delineation of the AVM nidus before GKR (Rojas-Villabona et al., 2017). After the acquisition of 3D GRE images using the protocol shown in Table $1 \mathrm{~b}$, a manual intra-venous bolus injection of $0.2 \mathrm{~mL} / \mathrm{kg}$ of Gadobenic acid $0.5 \mathrm{M}$ (MultiHance, Milan, Italy) was performed over approximately 4 seconds followed by a saline flush of $20 \mathrm{ml}$. The gad-T Theighted $^{-}$ and gad-TOF MRA protocols were respectively started approximately $1 \mathrm{~min}$ and $11 \mathrm{~min}$ after the gadolinium injection.

In addition to the imaging session, the pre-GKR patients underwent routine preoperative blood tests, including hematocrit measurements, which were performed at the same institution within three weeks before the MRI scan. 


\subsection{GRE Image Processing for QSM}

For both the 2D and 3D GRE data sets, a brain mask was calculated based on the first and last-TE magnitude images using the ITK-SNAP semi-automatic segmentation algorithm (Yushkevich et al., 2006). Based on the first-TE magnitude image, care was taken to include the superior sagittal sinus (SSS) in the brain mask to allow measurement of healthy vein oxygenation. Based on the seventh-TE magnitude image, care was taken to exclude areas of susceptibility-induced signal loss at longer TEs caused by the air-tissue interface near the paranasal sinuses and mastoid air cells. For further details on the calculation of the brain mask for QSM see Section S1.1 and Fig. S1 in Supplementary Material.

The processing pipeline for QSM included: temporal unwrapping and non-linear fitting (Liu et al., 2013) of the complex GRE signal over multiple echoes; spatial phase unwrapping using Laplacian kernels (Schweser et al., 2013); background field removal using Projection onto Dipole Fields (Liu et al., 2011); local field-to-susceptibility inversion using Tikhonov regularization (Kressler et al., 2010); correcting for the underestimation of the regularized inverse magnetic dipole (Schweser et al., 2013). For Tikhonov-based regularization and each GRE protocol (Table 1a and b), the optimal regularization parameter was calculated as the average across subjects of the individual optimal regularization parameters calculated using the L-curve method (Hansen and O'Leary, 1993). For further details on the choice of the local field-to-susceptibility inversion method and correction of $\chi$ underestimation see Section 1.2 and Fig. S2 in Supplementary Material.

For the subjects in which the transverse plane of the FoV was not perpendicular to $\mathbf{B}_{0}$, the following steps were performed before background field removal, to correct for the tilted acquisition (Dixon, 2018). First, the total field map was transformed from image to real world coordinates by resampling using the FSL Linear Image Registration Tool (FLIRT) (Jenkinson et al., 2012; Jenkinson and Smith, 2001) with spline interpolation (Dixon, 2018). The brain mask and the total field noise map calculated by the non-linear fit algorithm were also rotated using FLIRT with trilinear and spline interpolation, respectively.

QSM-based $\chi$ measurements are inherently relative because of the removal of background fields, thus they should be referenced to a tissue region whose susceptibility is not highly variable between subjects to enable the comparison of $\mathrm{SvO}_{2}$ across subjects. Here, each $\chi$ map was referenced to the posterior limb of the internal capsule (PLIC), which is a white matter region situated in the inferomedial part of each brain hemisphere. It has been shown (Straub et al., 2017) that $\chi$ measurements in the PLIC are nearly independent of age and have extremely small inter-subject variability. Thus, the PLIC was found to be the second-best region for $\chi$ referencing after the cerebrospinal fluid (CSF) in the lateral ventricles. The advantage of considering the PLIC instead of the CSF is that the former can be delineated in a semi-automated fashion based on an anatomical template (Lim et al., 2013). In contrast, for the CSF, manual segmentation is required to exclude the choroid plexus, a structure located within the ventricles and made of epithelial cells, loose connective tissue and capillaries.

For all patients and healthy subjects, the PLIC was automatically delineated based on the Eve susceptibility atlas (Lim et al., 2013). The Eve magnitude image was aligned to the skull-stripped fourth-echo magnitude image of all data sets, as the fourth TE was the closest to the TE of the Eve atlas $\left(\mathrm{TE}_{\mathrm{Eve}}=24 \mathrm{~ms}\right)$ in both the $3 \mathrm{D}\left(\mathrm{TE}_{4,3 \mathrm{D}}=26 \mathrm{~ms}\right)$ and $2 \mathrm{D}\left(\mathrm{TE}_{4,2 \mathrm{D}}=22.4 \mathrm{~ms}\right) \mathrm{GRE}$ acquisitions (Table 
1a and b). NiftyReg (Modat et al., 2010; Ourselin et al., 2001) was used to perform the coregistrations.

Susceptibility referencing was achieved by calculating the mean value of $\chi$ in the PLIC in both brain hemispheres and subtracting it from the $\chi$ map. In three pre-GKR patients (AVM04, AVM11 and AVM14), the PLIC of only one brain hemisphere was considered (left for AVM11 and right for AVM04 and AVM14), because in the contralateral hemisphere, the PLIC overlapped with the AVM or with paramagnetic hemosiderin deposits due to previous bleeding.

\subsection{Gad-TOF-MRA and Gad-T 1 -Weighted Image Processing}

In all the patients, both the gad-TOF MRA and gad- $\mathrm{T}_{1}$-weighted images were aligned with the firstecho GRE magnitude image using the affine registration algorithm in NiftyReg (Modat et al., 2010; Ourselin et al., 2001).

To improve visualization of the brain AVM, transverse maximum intensity projections (MIPs) of the coregistered gad-TOF MRA images were calculated for all the pre-GKR patients across slabs of five or ten slices for the 2D or 3D GRE images respectively.

\subsection{First-Echo GRE Magnitude Image Processing}

To investigate inflow effects, MIPs of the first-echo GRE magnitude image were calculated for all the patients across slabs of ten slices. In the first-echo magnitude images acquired using the 3D GRE acquisition, a TOF effect was expected to appear because of the short TE/TR. In the first-echo magnitude images acquired using the 2D GRE sequence, such a TOF effect was not expected to appear due to a combination of two factors: the longer TR values (i.e., 5636 ms compared with 46 ms for 3D GRE) and the use of a regional saturation technique (REST) saturation slab to suppress inflow effects. To confirm these hypotheses on the appearance of inflow effects, the MIPs of the first-echo GRE magnitude images were compared to the images acquired using the gad-TOF MRA sequence (Table 1c) and their MIPs.

\subsection{Calculation of Susceptibility-Based Venograms}

Susceptibility-based venograms of all patients and healthy subjects were calculated using probabilistic multiscale vessel filtering (MVF) (Bazin et al., 2016). This method was chosen because it had been specifically designed to segment vessel-like structures from $\chi$ maps.

Two parameters can lead to significant changes in the resulting vessel segmentation using MVF: the threshold value for the vessel probability map and the number of scales for the width of the Gaussian kernel. Because there was no a priori information on the vessel anatomy, the probability threshold was set to its default value (i.e., 0.5). The maximum number of scales for the smoothing Gaussian kernel determines the maximum detectable size for a vessel on the $\chi$ map. In a healthy subject, the average diameter of the largest cerebral veins is $4-5 \mathrm{~mm}$ (Durst et al., 2016). At the resolution of the 3D GRE protocol (i.e., $1.1 \mathrm{~mm}$ isotropic), assuming the absence of AVMs, large veins would be detected using four or more scales of the Gaussian kernel. Therefore, the optimal maximum number of scales was tested by applying MVF to the $\chi$ maps of all pre-GKR patients with a maximum number of scales ranging from 4 to 10 in integer increments. 
MVF with the parameters described above was applied to the $\chi$ maps of all the patients and the healthy volunteers. To segment the veins in the $\chi$ maps, the binary segmentation calculated using the MVF algorithm was multiplied by the corresponding $\chi$ map.

Transverse MIPs of the $\chi$ maps, vein segmentations and segmented $\chi$ maps were calculated to better appreciate and compare the vasculature. These MIPs were calculated across slabs of five or ten slices for 2D or 3D acquisitions respectively.

\subsection{Left and Right Brain Hemisphere Segmentation}

Masks for the left and right brain hemispheres (neurological convention) were created based on the Montréal Neurological Institute (MNI) International Consortium for Brain Mapping (ICBM) 2009c Nonlinear Symmetric Template with 1-mm isotropic resolution (Fonov et al., 2011; Fonov et al., 2009). The brain mask provided with this template did not include the SSS. Therefore, this structure was added to the brain mask using ITK-Snap (Yushkevich et al., 2006) with a combination of semiautomatic and manual segmentations. The resulting brain mask was then divided into left and right brain hemispheres by respectively isolating the regions to the left and right of the ICBM template's midline.

To enable the alignment of the left and right hemisphere masks to each subject's $\chi$ map, the gad- $\mathrm{T}_{1}$ weighted magnitude image of the ICBM template was aligned to the fourth-echo GRE magnitude image of each subject using NiftyReg. The fourth-echo GRE magnitude image was chosen as the reference image because it had a contrast between adjacent brain structures that was similar to the gad- $\mathrm{T}_{1}$-weighted magnitude image of the ICBM atlas. A combination of rigid and deformable transformations was used (Modat et al., 2010; Ourselin et al., 2001). The resulting spatial transformation was then applied to each hemispheric brain mask. Finally, to remove voxels outside the corresponding $\chi$ map, each coregistered hemispheric mask was multiplied by the same 3-voxel eroded brain mask used for QSM (Section 2.3).

\subsection{Calculation of Hemispheric Venous Densities}

Venous density was calculated in both brain hemispheres of all subjects as the ratio between the number of MVF-segmented "venous" voxels and the total number of voxels in the hemisphere. We aimed to assess if venous density could reflect the presence of a brain AVM. Therefore, in patients, venous density was compared in the hemisphere containing (pre-GKR) or once containing the AVM (post-GKR) and the contralateral hemisphere; in healthy volunteers, venous density was compared in the left and right hemispheres.

The mean and SD of venous density and the inter-hemispheric difference of the venous density's mean were calculated in all subjects and each hemisphere. Group-wise means and standard errors of the mean (SEMs) of the inter-hemispheric venous density difference were also calculated. The SEM was defined as the SD divided by the square root of the sample size, and it was chosen for group-wise analysis because it enabled comparing groups of different sizes.

In the pre-GKR patients, to investigate the origin of potential venous density alterations in the presence of an AVM, Spearman's correlation coefficient was calculated between the venous density in the hemisphere containing the AVM and the volume of the AVM nidus. For each pre-GKR patient, the volume of the AVM nidus was calculated based on gold-standard DSA images acquired for GKR 
planning and is reported in Inline Supplementary Table S1. The nidus volume was not available for one patient (AVM15), because they decided not to undergo GKR after DSA and MRI image acquisition. Therefore, in AVM15 the radiosurgical target was never delineated. Non-parametric Spearman's correlation was chosen due to the non-Gaussian distribution of the measured volumes and average venous densities. The significance of the correlation results was tested at a $5 \%$ significance level.

In all groups, the significance of the inter-hemispheric difference in venous density was tested using a Wilcoxon rank sum test. This test was chosen due to the non-Gaussian distribution of the venous density values across subjects and was performed with a $5 \%$ significance level. In the pre-GKR patients, it was tested if venous density was larger in the hemisphere containing the AVM than in the contralateral hemisphere (one-tailed test). In the post-GKR patients with a successful AVM obliteration, the draining veins are expected to disappear as the AVM obliterates. Therefore, in this group it was tested if venous density was similar in the hemisphere that contained the AVM before GKR and the contralateral hemisphere (two-tailed test), first including then excluding the patients with a residual AVM (i.e., GKR02, GKR04, GKR08, and GKR12). In the healthy volunteers, it was tested if venous density was similar in the left and right brain hemispheres (two-tailed test).

\subsection{Calculation of Hemispheric $\mathrm{SvO}_{2}$}

$\mathrm{SvO}_{2}$ was calculated for all subjects in each MVF-segmented voxel according to (Bazin et al., 2016; Cetin et al., 2016; Fan et al., 2014; Fan et al., 2016; Ozbay et al., 2015; Serres et al., 2015; Weisskoff and Kiihne, 1992; Xu et al., 2014):

$$
\mathrm{SvO}_{2}=1-\frac{\Delta \chi_{\text {vein-water }}-\Delta \chi_{\text {oxy-water }} \mathrm{Hct}}{\Delta \chi_{\text {do }} \mathrm{Hct}}
$$

where $\Delta \chi_{\text {vein-water }}$ is the $\chi$ shift between venous blood and water measured using QSM, $\Delta \chi_{d o}=$ $0.27 \cdot 4 \pi \mathrm{ppm}$ (Spees et al., 2001) the $\chi$ shift per unit hematocrit (Hct) between fully deoxygenated and fully oxygenated red blood cells, and $\Delta \chi_{\text {oxy-water }}=-0.03 \cdot 4 \pi \mathrm{ppm}$ (Weisskoff and Kiihne, 1992) the $\chi$ shift per unit Hct between oxygenated red blood cells and water.

In the pre-GKR patients, the individual measures of hematocrit (see Inline Supplementary Table S1) were used and as expected they were within normal limits. For subjects in which hematocrit measurements were not available (two pre-GKR patients and the whole post-GKR and healthy volunteer groups), a hematocrit value of 0.4 was assumed.

For each subject, the mean and $\mathrm{SD}$ of $\mathrm{SvO}_{2}$ were calculated in each hemisphere. In addition, group means and $\mathrm{SDs}$ of $\mathrm{SvO}_{2}$ were calculated in the hemisphere with and without the AVM (patients) or in the left and right hemisphere (healthy volunteers).

In the pre-GKR patients, to investigate the hemispheric distribution of $\mathrm{SvO}_{2}$, a histogram was calculated of the hemispheric $\mathrm{SvO}_{2}$ values in the MVF-segmented voxels. $\mathrm{SvO}_{2}$ values outside the 0 $100 \%$ range, which were mathematically admissible as a solution of Eq. 1 but non-physiological, were excluded from this analysis. Each histogram was fitted using a kernel smoothing function (for further details see Section S1.3 in Supplementary Material), and the local maxima of the fitted curve were calculated. If an AVM's draining veins were correctly segmented in the venogram, a bimodal distribution of $\mathrm{SVO}_{2}$ was expected in the hemisphere containing the brain $\mathrm{AVM}$, with the primary peak centered around the average $\mathrm{SvO}_{2}$ of healthy veins and a secondary peak centered around a 
higher value of $\mathrm{SvO}_{2}$ measured in the AVM draining veins. In contrast, the $\mathrm{SvO}_{2}$ distribution in the hemisphere without the AVM was expected to only have one mode centered around the $\mathrm{SvO}_{2}$ value of healthy veins.

Similar to the venous density analysis, in all groups the significance of the inter-hemispheric difference in $\mathrm{SvO} 2$ was tested using a non-parametric Wilcoxon rank sum test with a $5 \%$ significance level. In the pre-GKR patients, it was tested if $\mathrm{SvO}_{2}$ was larger in the hemisphere containing the AVM than in the contralateral hemisphere (one-tailed test). In the post-GKR patients, it was tested if $\mathrm{SvO}_{2}$ was similar in the hemisphere that contained the AVM before GKR and in the contralateral hemisphere (two-tailed test) excluding the patients with a residual AVM (i.e., GKR02, GKR04, GKR08, and GKR12). In the healthy volunteers, it was tested if $\mathrm{SvO}_{2}$ was similar in the left and right brain hemispheres (two-tailed test).

\subsection{Selection of ROIs for Calculation of $\mathrm{SvO}_{2}$ in the AVM Draining Veins}

For each pre-GKR patient, three regions of interest (ROIs) were identified and drawn using ITKSNAP's active contour semi-automatic segmentation (Yushkevich et al., 2006).

One ROI ( $\left.R O I_{D V}\right)$ was drawn on a vein directly draining the AVM. Such a draining vein was identified on the gad-TOF MRA image in consultation with a radiology clinical fellow (ARV) as the largest blood vessel linking the AVM nidus to a major venous drainage structure such as the venous sinuses or internal cerebral veins. $\mathrm{ROI}_{D V}$ was delineated on the initial segment of the draining vein just after it left the AVM nidus. In one case, where the anatomy of the AVM's venous drainage was unclear from the gad-TOF MRA, the DSA image was reviewed by ARV to understand the draining pattern and confirm the location of the draining vein. ROIDV was drawn on the gad-TOF MRA image and had a different size for each patient due to the variable anatomy of the AVMs.

Another ROI (ROIsss) was drawn on a region of the SSS that did not drain blood from the AVM, and, therefore, could be regarded as healthy. This 'upstream' portion of the SSS was drawn on the gad- $\mathrm{T}_{1}$ weighted image, as, depending on the location of the AVM, the gad-TOF MRA image did not always include the SSS. For each patient, $\mathrm{ROI}_{\text {sss }}$ was drawn on different locations of the SSS to ensure the exclusion of regions draining blood from the AVM. To provide a baseline $\mathrm{SvO}_{2}$ measurement in healthy veins while reducing the effect of partial voluming, for thirteen pre-GKR patients a third ROI ( ROI $_{\text {Healthyvein2) }}$ was drawn on the gad- $\mathrm{T}_{1}$-weighted image in a healthy vein other than the SSS (for details, see Section S1.4 in Supplementary Material).

All ROIs were aligned with the $\chi$ map using the gad-TOF MRA and gad-T ${ }_{1}$-weighted to GRE image transformations calculated previously (Section 2.4). $\mathrm{SvO}_{2}$ was measured in each voxel of all ROls using Eq. (1) with the same values for $\Delta \chi_{d o}, \Delta \chi_{\text {oxy-water }}$ and Hct described in Section 2.9. In each patient, the mean and SD of $\mathrm{SvO}_{2}$ were calculated in all ROIs. Moreover, the means and SEMs of $\mathrm{SvO}_{2}$ in all ROIs were calculated across patients. As the three venous ROls had different sizes in individual patients, the SEM was chosen instead of the SD to enable the comparison of the variability of $\mathrm{SvO}_{2}$ across patients. Using a one-tailed Wilcoxon rank sum test (significance level $=5 \%$ ), it was then tested if $\mathrm{SvO}_{2}$ in $\mathrm{ROI}_{\mathrm{DV}}$ was larger than in $\mathrm{ROI}_{\text {sss. }}$. For further details on the calculation of a baseline $\mathrm{SvO}_{2}$ value in healthy veins and its comparison with an $\mathrm{SvO}_{2}$ value in draining veins see Section S1.4 in Supplementary Material. 


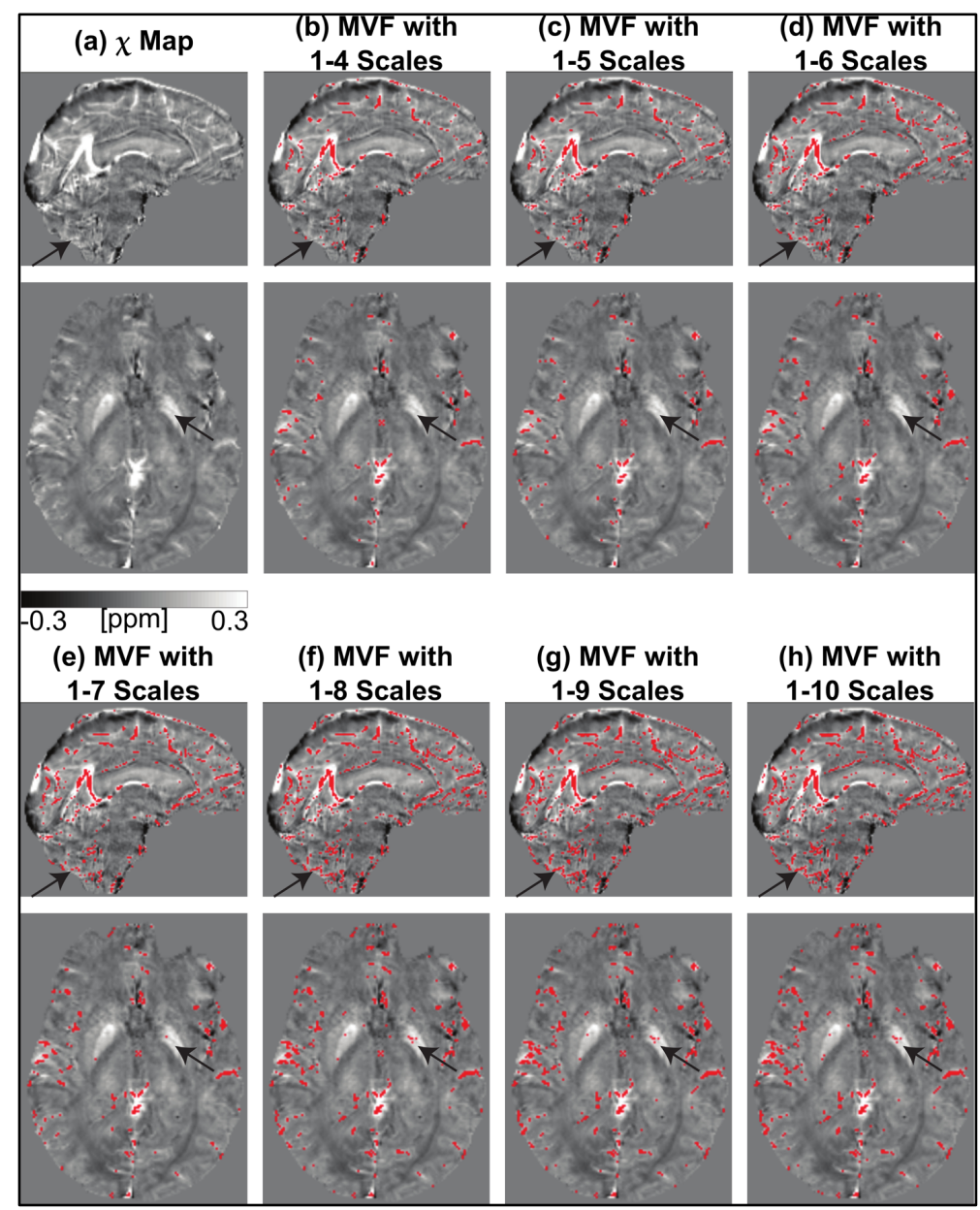

Fig. 1 MVF segmentations for an increasing maximum number of scales. For a representative pre-GKR AVM patient, a sagittal and an axial slice of the $\chi$ map (a) and the corresponding MVF segmentations are shown for an increasing maximum number of Gaussian filter scales (b-f). The arrows point at regions where the MVF segmentation was inaccurate for higher values of the maximum number of Gaussian filter scales.

\section{Results}

\subsection{Parameter Optimization for Tikhonov-Based Regularization}

In the subjects scanned using the 3D and 2D GRE protocols (Table 1a and b), the average optimal regularization parameters for Tikhonov-based regularization were respectively equal to 0.0684 and 0.0547 .

\subsection{Parameter Optimization for Multiscale Vessel Filtering}

Fig. 1 shows the $\chi$ map and the corresponding MVF segmentations of a representative pre-GKR patient with a small AVM in the cerebellum (not shown in these image slices). Because of the lack of a ground truth map of the venous vasculature, the accuracy of the segmentation could not be quantified. However, it was noted that on increasing the maximum number of scales the segmentation appeared increasingly noisy (Fig. 1, arrows on the sagittal slice). Moreover, on increasing the maximum number of scales, non-venous voxels were included into the final vein segmentation image, for example, in the paramagnetic deep gray matter nuclei (Fig. 1, arrows on the transverse slice). At the same time, increasing the number of maximum scales did not visibly improve the segmentation of larger veins as would be expected. Therefore, to minimize noise, it was 
decided to apply the algorithm using a maximum of 4 scales for the Gaussian kernel, which corresponded to a maximum detectable vessel diameter of $4.4 \mathrm{~mm}$ along each imaging axis.

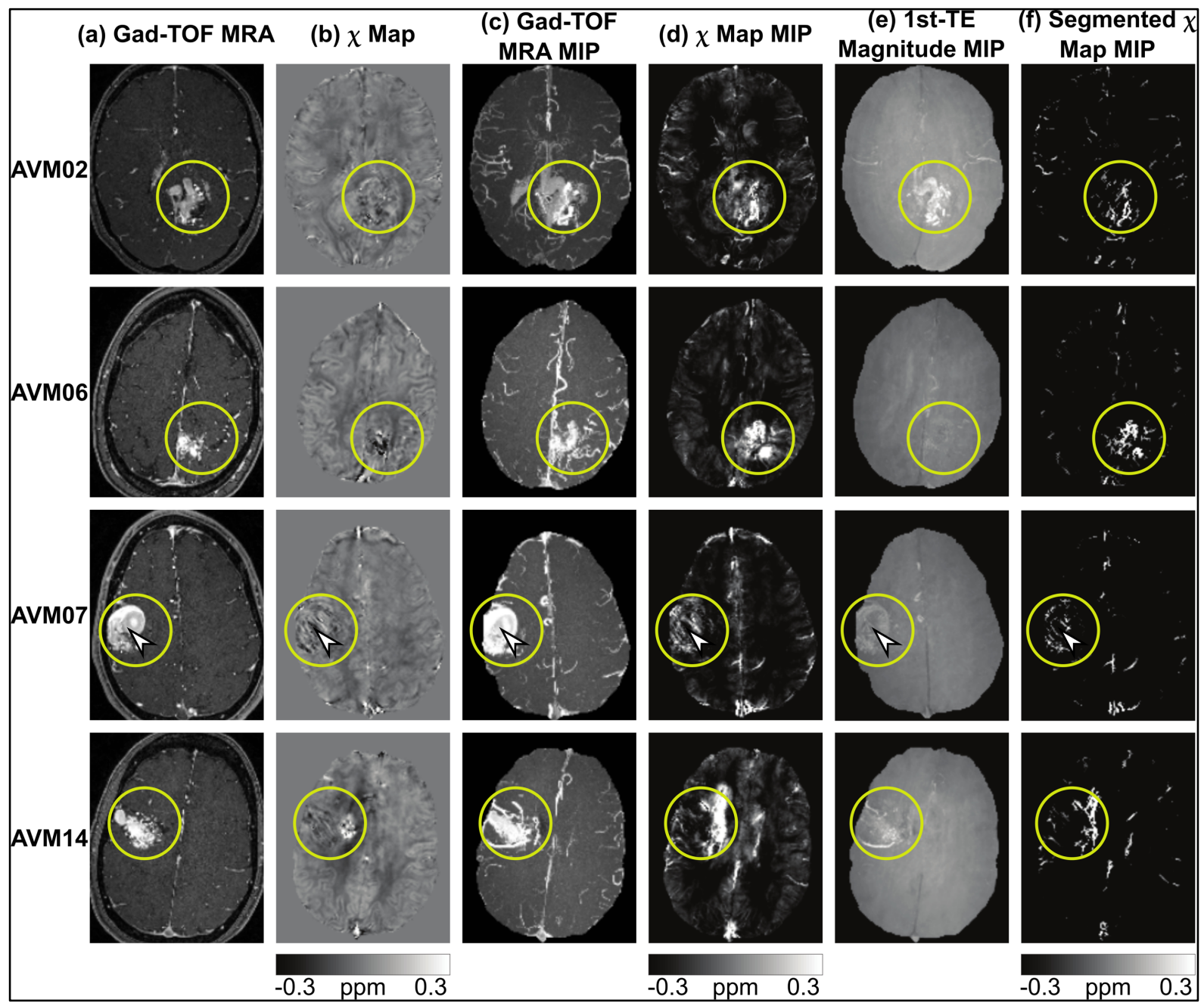

Fig. 2 Multimodal imaging appearance of brain AVMs in four representative pre-GKR patients. The figure shows the same transverse slice of the coregistered gad-TOF MRA image (a) and the $\chi$ map (b) and the same transverse slice (in a location similar to (a) and (b) of the MIP of the coregistered gad-TOF MRA (c), the MIP of the $\chi$ map (d), the MIP of the first-TE GRE magnitude image (e) and the $\chi$ map segmented using MVF (f). AVMs are circled in yellow. In subject AVMO7, the white arrowhead points at an arterial structure in the AVM, whose presence was detected on the $\chi$ map. All images in columns (a), (c) and (e) are in arbitrary units and, in each column, the images are scaled within the same range.

\subsection{AVM Detection}

Fig. 2 shows that the features of a brain AVM are clearly identifiable in both the gad-TOF MRA images and MIPs (Figs. 2a and c); $\chi$ maps and MIPs (Figs. 2b and d); and, to a lesser extent, in the first-TE GRE magnitude image (Fig. 2e).

Fig. 3 shows that the previous presence of a brain AVM was detectable after GKR in three patients (GKR05, GKR06 and GKR13). The MIP of the first-echo GRE magnitude acquired using the 2D sequence did not contain TOF effects, which was expected because of the use of a REST slab the longer TR compared to the 3D sequence (Table 1). In contrast with the pre-GKR patients, the firstecho GRE magnitude MIP in the post-GKR patients did not show bright AVM features (subjects GKR13 and GKR17 in Fig. 3e). 
Notably, in both Figs. 2 and 3, some relatively large areas around the AVM appeared bright in the $\chi$ map (Figs. 3b and d) but not in the gad-TOF MRA image (Figs. 3a and c). Examples include the area circled in the images of patients AVM14 (fourth row in Figs. 2a-d) and GKR06 and GKR13 (fourth row in Figs. 3a-d). Bleeding can occur in the presence of an AVM due to rupture of the vessels caused by high blood pressure. Therefore, it is possible that these regions appeared bright only on the $\chi$ images because they contained paramagnetic hemosiderin deposits (Duyn and Schenck, 2017) from a previous bleed.

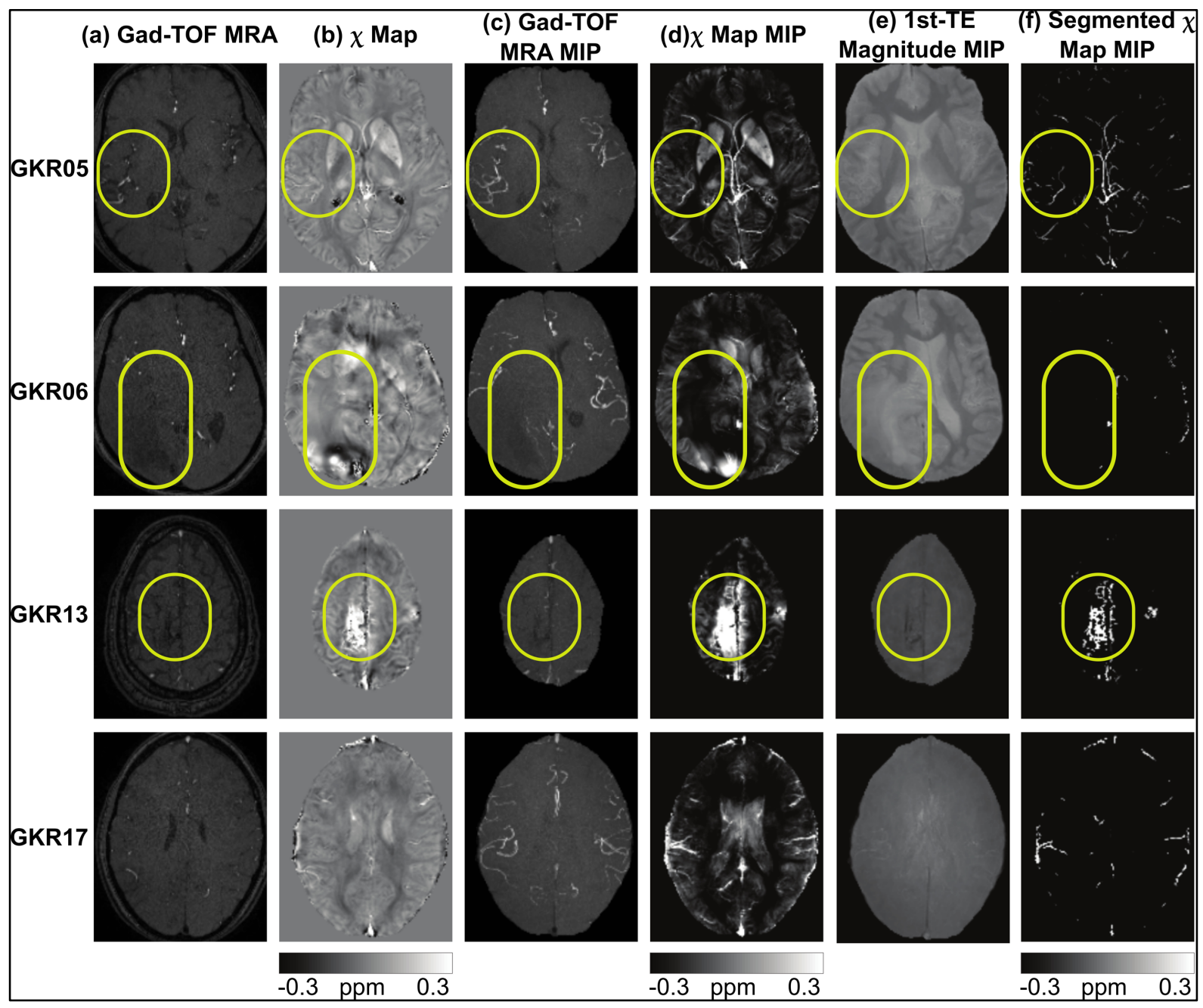

Fig. 3 Multimodal imaging appearance of brain AVMs in four representative post-GKR patients. The figure shows the same transverse slice of the coregistered gad-TOF MRA image (a) and the $\chi$ map (b) and the same transverse slice (in a location similar to (a) and (b)) of the MIP of the coregistered gad-TOF MRA (c), the MIP of the $\chi$ map (d), the MIP of the first-TE GRE magnitude image (e) and the $\chi$ map segmented using MVF ( $f$ ). When identifiable, the area previously containing the AVM is circled in yellow. For QSM, patients GKRO5 and GKRO6 were scanned using the 2D GRE sequence, whereas patients GKR13 and GKR17 were scanned using the 3D GRE sequence. All images in columns (a), (c) and (e) are in arbitrary units and, in each of these columns, the images are scaled within the same range. To allow direct comparison of the pre-GKR (Fig. 2) and post-GKR results, the gad-TOF MRA images (a) are scaled to the same range of values as in Fig. $2 a$.

\subsection{Hemispheric Venous Density}

In the pre-GKR patients (Fig. 4a), the hemisphere containing the AVM had the largest venous density in all patients except patient AVM12, who had a small AVM draining pattern (see size of ROIDV in Inline Supplementary Table S3). The mean and SD of the venous density across patients were both larger in the AVM-containing hemisphere $(1.43 \pm 0.45 \%)$ than in the hemisphere without the AVM 
$(1.15 \pm 0.27 \%)$. The mean venous density was found to be significantly larger in the AVM-containing hemisphere than in the contralateral hemisphere $(p=0.03)$.

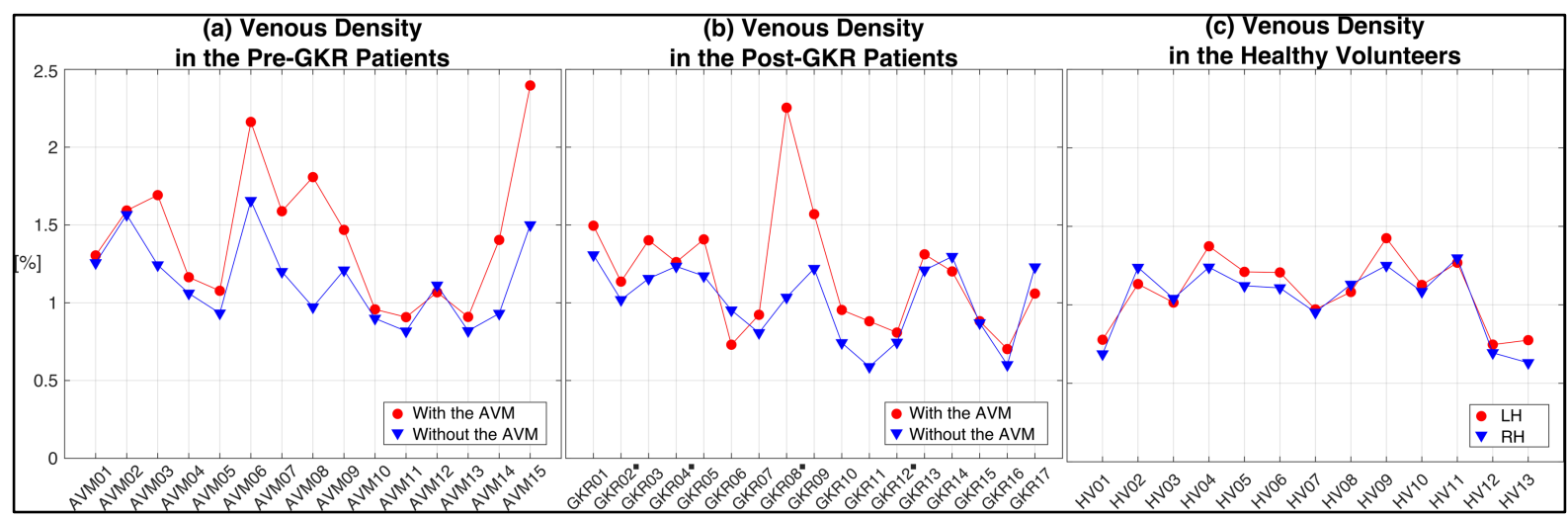

Fig. 4 Hemispheric venous densities in the pre-GKR (a) and post-GKR patients (b) and healthy volunteers (c). Venous density values are shown for brain hemispheres with and without the brain $\operatorname{AVM}(a, b)$ and for the left and right hemispheres (c). In (b), the patient tags with a black diamond symbol denote the presence of residual AVM after GKR.

In the post-GKR patients (Fig. 4b), the hemisphere that previously contained the AVM often had the largest venous density. Exceptions were observed in patients GKR06, GKR14 and GKR17. In line with the results of the pre-GKR patients, in the post-GKR patients with a residual AVM (i.e., GKR02, GKR04, GKR08 and GKR12) the hemisphere containing the AVM always had the largest venous density.

In the post-GKR patients (Fig. 4b), the mean and SD of the venous density across all patients were slightly larger in the hemisphere that contained the AVM before GKR $(1.18 \pm 0.39 \%)$ than in the contralateral hemisphere $(1.01 \pm 0.24 \%)$. This same relationship was also observed excluding the four patients with residual AVMs, with slight changes in the means and SDs of the venous density in both the hemisphere that contained the AVM before GKR $(1.12 \pm 0.30 \%)$ and contralateral hemisphere $(1.01 \pm 0.26 \%)$. However, these hemispheric differences in venous density were not significant.

In the healthy volunteers (Fig. 4a), the venous density had similar means and SDs in the left (1.09 \pm $0.22 \%)$ and right $(1.04 \pm 0.23 \%)$ hemispheres. Here, no significant difference was found between the venous density of the two brain hemispheres.

The means and SEMs of the inter-hemispheric difference of venous density were larger in both the pre-GKR $(0.29 \pm 0.07 \%)$ and post-GKR patients $(0.22 \pm 0.07 \%$ or, excluding patients with a residual $A V M, 0.18 \pm 0.03 \%)$ than in the healthy volunteers $(0.08 \pm 0.01 \%)$. In the post-GKR patients with a residual AVM, the inter-hemispheric difference of venous density (mean and SEM: $0.36 \pm 0.29 \%$ ) was more similar to the corresponding value measured in the pre-GKR patients than in the post-GKR patients with successful GKR.

According to the Wilcoxon rank sum test, the means of the inter-hemispheric difference of venous density were significantly different in both the pre-GKR $(p=0.048)$ and post-GKR patients $(p=0.01$ both including and excluding the subjects with a residual AVM) compared to the healthy volunteers. 


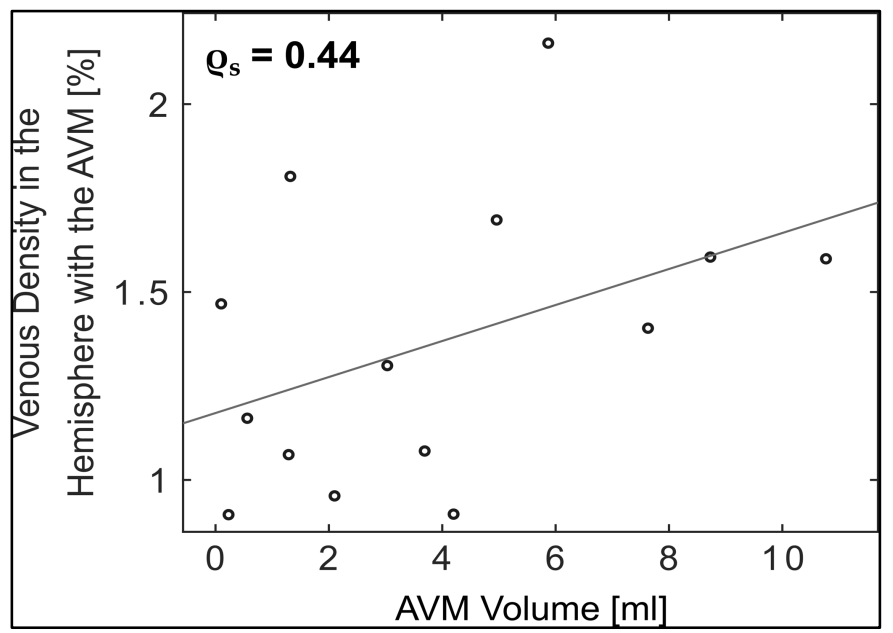

Fig. 5 Correlation between the AVM's volume and the venous density in the brain hemisphere with the AVM in the pre-GKR patients. The correlation coefficient is reported on the top left corner of the scatter plot.

A weak positive linear correlation (Fig. 5 , correlation coefficient $\rho_{S}=0.44$ ) was found between the volume of the AVM nidus and the venous density of the hemisphere containing the AVM in the preGKR patients. However, the correlation between these two variables was not significant.

\subsection{Hemispheric $\mathrm{SvO}_{2}$}

For the referencing of the $\chi$ maps, the mean \pm SD of the $\chi$ in the PLIC across all subjects was $-0.01 \pm$ $0.02 \mathrm{ppm}$.

In contrast with the hemispheric venous density results (Fig. 4), in the patients the hemispheric $\mathrm{SvO}_{2}$ was not consistently larger in the hemisphere containing the AVM both before (see Inline Supplementary Fig. S3a) and after GKR (see Inline Supplementary Fig. S3b). As a result, no significant inter-hemispheric $\mathrm{SvO}_{2}$ difference was found in patients or healthy volunteers. Moreover, in both brain hemispheres, no significant difference was found between the hemispheric $\mathrm{SvO}_{2}$ in the AVM patients compared to the healthy volunteers.

In the histogram analysis, only one meaningful local maximum of $\mathrm{SvO}_{2}$ was detected and it was always in the $70-80 \%$ range. If more local peaks were detected, these included only a few voxels with a much lower value of $\mathrm{SvO}_{2}$, e.g., 17\% (see Inline Supplementary Fig. S4a). However, such small values of $\mathrm{SvO}_{2}$ are non-physiological, as gold-standard positron emission tomography (PET) measurements report $\mathrm{SvO}_{2}$ values around 60\% (Bremmer et al., 2011; Hattori et al., 2004).

Therefore, additional local maxima in the histograms did not indicate the presence of an AVM. Their presence was rather linked to noise in the hemispheric $\mathrm{SvO}_{2}$ distributions. Similar results were observed in the healthy volunteers and in the post-GKR patients.

For further details on the hemispheric $\mathrm{SvO}_{2}$ results, see Section S2.1 in Supplementary Material.

\subsection{ROI-Based $\mathrm{SvO}_{2}$}

In the pre-GKR patients, the size of $\mathrm{ROI}_{\text {sss }}$ and $\mathrm{ROI}_{\mathrm{DV}}$ varied across a considerably large range (see Inline Supplementary Table S3), reflecting the highly variable anatomy and location of brain AVMs across patients. For the calculation of ROI-based $\mathrm{SvO}_{2}, 23 \pm 19$ and $33 \pm 64$ voxels were respectively 
removed from $\mathrm{ROI}_{\text {sss }}$ and $\mathrm{ROI}_{\mathrm{Dv}}$ and because their corresponding $\mathrm{SvO}_{2}$ value was outside the $0-100 \%$ range.

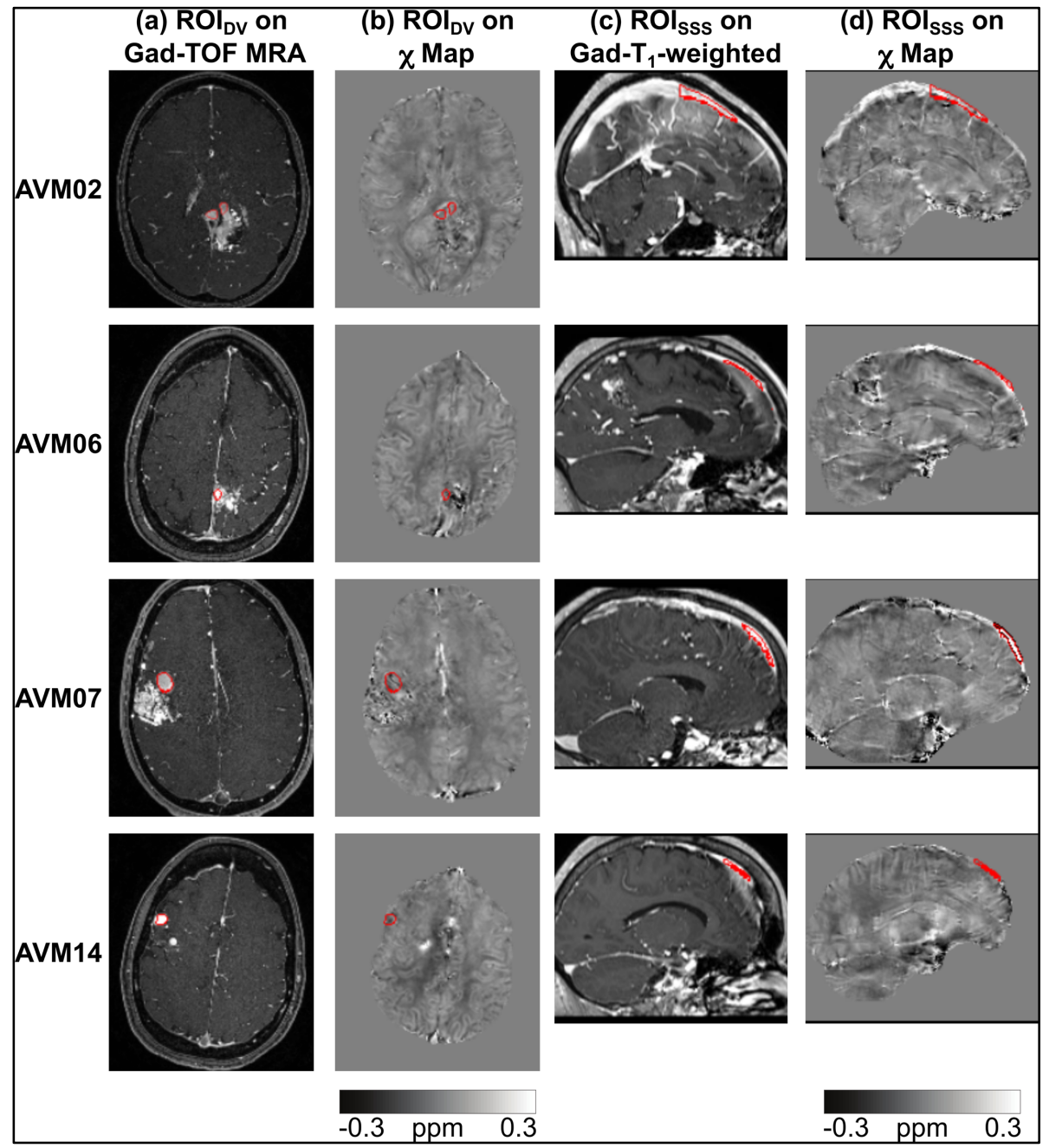

Fig. 6 AVM draining vein and SSS ROIs in four representative pre-GKR patients. The contour of the AVM draining vein ROI is shown overlaid on the same transverse slice of the gad-TOF MRA image (coregistered to the $\chi$ map) (a) and the $\chi$ map (b). Similarly, the contour of the SSS ROI is shown overlaid on the same sagittal slice of the gad- $T_{1}$-weighted image (coregistered to the $\chi$ map) (c) and the $\chi$ map (d). All images in columns (a) and (c) are in arbitrary units and, in each of these columns, the images are scaled within the same range. These subjects are the same as those shown in Fig. 2: for patients AVM02 and AVM06 the same transverse slice is shown as in Fig. 2; for patients AVM07 and AVM14 a different transverse slice was chosen as the one shown in Fig. 2 did not include the draining vein ROI.

ROIDv always delineated an area with a similar $\chi$ to the surrounding brain tissue on the $\chi$ maps (Fig. $6 \mathrm{~b})$. In most pre-GKR patients, the region delineated by ROIsss appeared bright on the $\chi$ map (AVM02, AVM06 and AVM07 in Fig. 6d). However, in four pre-GKR patients (AVM06, AVM08, AVM14 in Fig. $6 \mathrm{~d}$ and AVM15), the region delineated by $\mathrm{ROI}_{\text {sss }}$ had a $\chi$ similar to the surrounding brain tissue. 


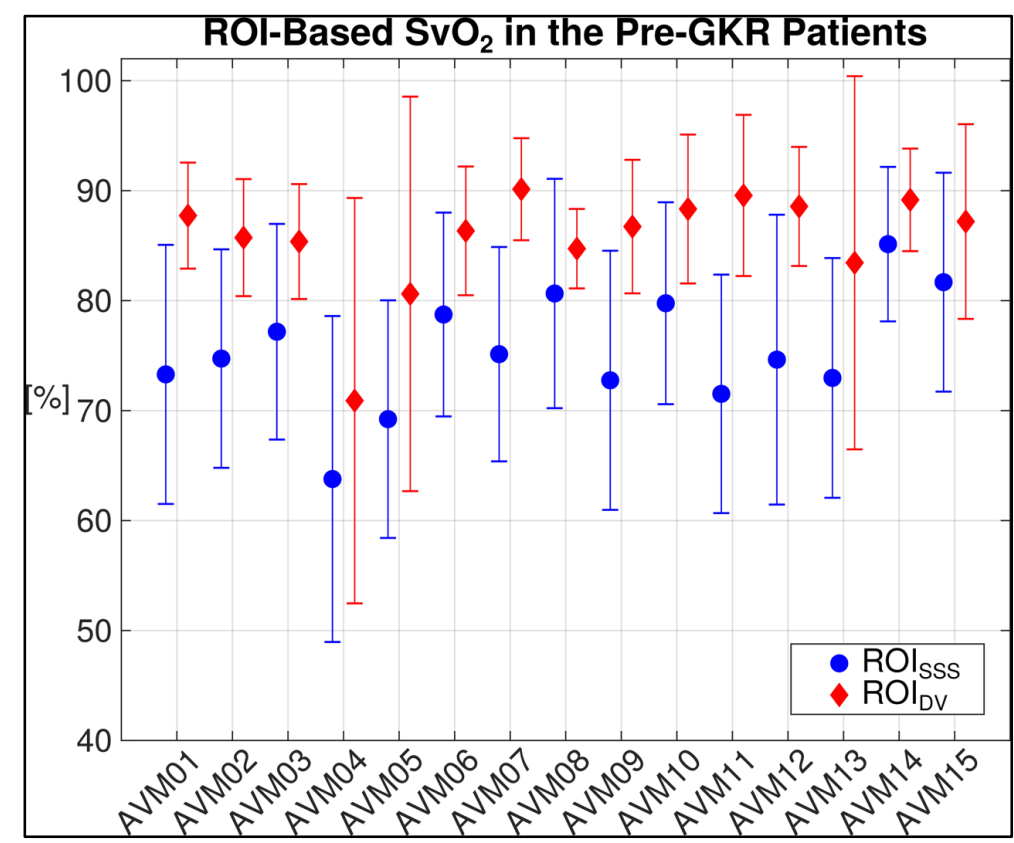

Fig. $7 \mathrm{ROI}$-based $\mathrm{SvO}_{2}$ measurements in the pre-GKR patients. For the pre-GKR patients, the figure shows the mean $\mathrm{SvO}_{2}$ values measured in two ROIs: one drawn on the superior sagittal sinus (ROIsss) and the other on the largest vein draining the AVM (ROIDV). The error bars denote the SD of the measurements.

$\mathrm{SvO}_{2}$ was always larger in $\mathrm{ROI}_{\mathrm{DV}}$ than in $\mathrm{ROI}_{\text {sss }}$ (Fig. 7). The same relationship was also observed at a group level, as the means and $\mathrm{SEMs}$ of $\mathrm{SvO}_{2}$ across patients were equal to $75.41 \pm 1.38 \%$ and $85.63 \pm$ $1.24 \%$ in $\mathrm{ROI}_{S S S}$ and $\mathrm{ROI}_{\mathrm{DV}}$ respectively. $\mathrm{SvO}_{2}$ was found to be significantly higher in $\mathrm{ROI}_{\mathrm{DV}}$ than in $\mathrm{ROI}_{\text {sss }}(\mathrm{p}<0.001)$. For further details on the $\mathrm{SvO}_{2}$ results in $\mathrm{ROI}_{\text {Healthyvein2 }}$ see Section $\mathrm{S} 2.2$ in Supplementary Material.

\section{Discussion}

This study aimed to evaluate the appearance of arteriovenous malformations (AVMs) on MRIderived quantitative susceptibility $(\chi)$ maps, and compare venous vascularization and oxygenation in the brains of patients with an AVM before and after gamma knife radiosurgery (GKR) with healthy volunteers. In the QSM protocol, comparison of distinct strategies for performing local field-tosusceptibility inversion enabled the calculation of $\chi$ maps in which streaking artifacts were minimized. Therefore, streaking artifacts were not expected to significantly affect $\chi$ measurements. In the pre-GKR patients, comparing $\chi$ maps with gad-TOF MRA images acquired for high-resolution depiction of the AVM enabled evaluation of the AVM features appearing in $\chi$ maps. This comparison also enabled investigation of whether the venous drainage of a brain AVM could be identified using $\chi$-based vein segmentation. Similarly, in the post-GKR patients, comparing $\chi$ maps with gad-TOF MRA images of the brain area containing the AVM before GKR allowed us to investigate whether scar tissue, paramagnetic hemosiderin deposits due to a previous hemorrhage, or residual AVM features could be detected on the $\chi$ maps and how these might affect $\chi$-based vein segmentation. To better explore these relationships, hemispheric venous density and oxygenation $\left(\mathrm{SvO}_{2}\right)$ values were calculated using $\chi$-based vein segmentations in AVM patients before and after treatment with $\mathrm{GKR}$, and in healthy volunteers. Finally, in the pre-GKR patients, the comparison of $\mathrm{SvO}_{2}$ values in two venous ROIs allowed testing of the hypothesis that mixed arterial and venous blood draining from the AVM leads to increased $\mathrm{SvO}_{2}$ compared to healthy veins. 
The AVM's feeding arteries and nidus were not expected to appear in the susceptibility maps, because arterial blood and the surrounding brain parenchyma have a similar diamagnetic susceptibility. Moreover, in the pre-GKR patients the MRA-derived AVM nidus volume did not significantly correlate with the venous density in the hemisphere containing the malformation. Therefore, regions highlighted in the gad-TOF MRA images (Figs. 2a and $c$ ) and appearing bright in the $\chi$ maps (Figs. $2 b$ and d) could be AVM draining veins. However, the shape of the AVM features in the segmented $\chi$ maps was dissimilar to the shape of the AVM veins visible in the gad-TOF MRA images (Figs. 2c, d and f). For example, the vessel highlighted on the gad-TOF MRA image of patient AVM07 (arrowhead on the gad-TOF image of patient AVM07 in Fig. 2a) was an artery but it was detectable on the $\chi$ map (arrowhead on the $\chi$ map of patient AVM07 in Fig. 2b). Therefore, susceptibility-based MVF segmentation of brain AVMs may have been influenced by additional factors. One confounder could be GRE signal dephasing induced by tortuous blood flow within the vessels in and around the AVM. For example, in patient AVM07 flow-induced dephasing inside an arterial structure (arrowhead on the gad-TOF image in Fig. 2b) and appearing bright on the $\chi$ map (Fig. 2d) was erroneously segmented as venous by the MVF algorithm (Fig. 2f). The lack of flow compensation could have also contributed to decreasing the visibility of AVM draining veins, because in non-flow-compensated images veins usually appear displaced between echoes due to incorrect phase encoding of the GRE signal. On the other hand, the nonlinear fitting method used to combine multiple TEs may have mitigated potential errors due to flow effects. Indeed, this method assigns smaller weights to unexpected signal changes and regions of low signal-to-noise ratio, and has been shown to improve QSM image quality in both healthy veins and intracerebral hemorrhage (Liu et al., 2013). In some cases, bright regions on the $\chi$ map could also be due to paramagnetic hemosiderin deposits from past bleeding or could be artifacts caused by a previous embolizing treatment. For example, the bright elongated shape on the $\chi$ map of patient AVM14 (Figs. $2 \mathrm{~d}$ and f) may have appeared because of the $\chi$ difference between brain tissue and an area previously treated using embolizing glue. There are several embolizing agents available for the endovascular treatment of brain AVMs. One of the most frequently used is called Onyx (Medtronic Neurovascular, Irvine, CA, USA) and consists of ethylene vinyl alcohol copolymer, dimethyl-sulfoxide and tantalum powder (Vollherbst et al., 2018). Tantalum is a paramagnetic metal (Schenck, 1996) used to obtain radiopacity of the glue during treatment but also causing susceptibility artifacts on post-operative MRI (Cronqvist et al., 2006; Vollherbst et al., 2018). To avoid this limitation, new embolizing agents are being tested in which tantalum is replaced by other non-metallic radiopaque materials (Vollherbst et al., 2018). Therefore, in the future QSM of embolized brain AVMs could be unaffected by glue-induced $\chi$ artifacts.

In the pre-GKR patients, the AVM features appearing on the first-echo GRE magnitude image often reflected the shape of some AVM features appearing on the gad-TOF MRA (patients AVM02, AVM07 and AVM14 in Fig. 2e). As the TOF effect highlights fast-flowing blood in both the 3D GRE first-TE magnitude image (Fig. 2e) and the MRA images (Figs. 2a and c) the regions appearing bright in both of these images were probably AVM feeding arteries or vessels in the AVM nidus expected to contain fast-flowing arterial blood. These results also demonstrate that GRE sequences for QSM can be tuned to obtain a TOF-sensitive contrast and therefore used for the simultaneous detection of arteries and veins.

In the post-GKR patients, altered vessel anatomies were not detected in the first-TE 3D GRE magnitude image or in the MVF segmentation of the susceptibility maps because the nidus of an 
AVM disappears following successful GKR (Fig. 3). Moreover, scar tissue due to AVM obliteration did not appear in the MVF segmentations indicating that the MVF algorithm could not identify features in the $\chi$ image that would distinguish between scar tissue and the surrounding healthy brain tissue.

Studies on automatic vein segmentation of SWI images (Beriault et al., 2015; Ward et al., 2018) have addressed the issue of potential false positives in the deep gray matter nuclei by incorporating anatomical templates of the basal ganglia as segmentation priors. Instead, the MVF algorithm applies a recursive ridge filter followed by a global probability diffusion approach, which enhances the filter response along connected components while removing unconnected ones. By tuning the parameters of the MVF algorithm, we effectively minimized the chance of false positives in paramagnetic deep grey matter regions (see Fig. 1). Past bleeding of an AVM represents an additional potential source of false positives in the MVF venograms, which was not explicitly addressed in this study. Instead, because hemorrhage linked to an AVM occurs around the malformation, false positives due to past bleeding were considered as a confounder potentially increasing the total venous density in the same hemisphere as the AVM. In this sense, hemorrhage represents a characteristic that may aid in the automatic identification of vascular malformations based on inter-hemispheric differences in venous density. Future work could explore the minimization of false positives due to hemorrhage in the MVF segmentation. This issue could be addressed by delineating and excluding areas of past bleeding from the $\chi$ map before the application of MVF. It must be noted that such an approach would add a manual step to the otherwise automatic MVF algorithm, thus making the procedure for vein density estimation semiautomatic.

$\chi$-based venograms of pre-GKR patients revealed a significant increase in venous density in the hemisphere containing the brain AVM (Fig. 4a) compared with the contralateral hemisphere. A similar but not significant increase in venous density was also observed in post-GKR patients (Fig. 4b), whereas no increase was observed in healthy volunteers (Fig. 4c). The increased venous density in the patients indicates that the current or past presence of an AVM (and potential bleeding episodes associated to it) create ridges on the $\chi$ map that are detectable using MVF. In the pre-GKR patients, a significant increase in the number of ridges on the $\chi$ map could reflect the higher vascularization of AVMs compared to healthy tissue. AVM neovascularization is a dynamic process that occurs in response to hypoxia of the surrounding tissue and leads to AVM growth over time mainly via vasculogenesis, i.e., de novo formation of new vasculature (Lu et al., 2011). Ridges on the $\chi$ maps of pre-GKR patients could also stem from confounding factors such as fast-flowing blood in the AVM's arteries and hemosiderin deposits of a past hemorrhage in and around the AVM. Both of these factors were discussed earlier in this section. Because AVMs are expected to disappear after successful treatment, in the post-GKR patients, ridges on the $\chi$ map could be caused by hemosiderin deposits from past bleeding or by previous embolizing treatment. The paramagnetic characteristics of embolizing agents were discussed earlier in this section. The significance of increased venous density only in untreated AVMs suggests that segmentation of susceptibility maps using MVF might offer valuable information for automated detection of brain vascular malformations. To facilitate this, it is recommended to set the size of the Gaussian smoothing filter to detect the largest diameter of healthy veins, as larger filter sizes led to more false positives in the MVF segmentations (Fig. 1) and the largest AVM draining veins were invisible on the $\chi$ maps (Figs. 6a and b). The calculation of $\chi$-based venous densities could be implemented as a screening tool for the automatic detection of vascular malformations in people undergoing MRI of the brain. In fact, incidental 
findings of brain AVMs or cavernous malformations on MRI are relatively common with a prevalence of $0.05 \%$ and $0.16 \%$, respectively (Morris et al., 2009).

In the pre-GKR patients, the largest draining vein of an AVM segmented on the gad-TOF MRA image did not appear bright in the corresponding coregistered $\chi$ map (Fig. 6) most likely because of the high oxygenation of blood in these vessels. Therefore, the veins draining an AVM should be

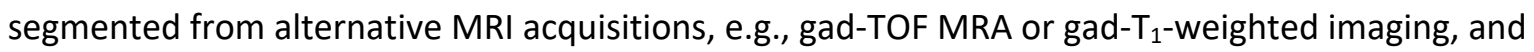
the segmented vessels should be then coregistered to the image space of the $\chi$ map. Such a segmentation could be facilitated by the use of a digital atlas of the venous cerebrovasculature. Such an atlas could also enable the automatic detection of anomalous vein anatomies based on MRA or $\chi$-based images. Some studies have developed digital atlases of the brain vasculature including either the arteries (Dunas et al., 2017) or the veins (Ward et al., 2018). Others have developed such digital atlases including both the arteries and the veins with (Bernier et al., 2018; Huck et al., 2018) or without (Passat et al., 2005; Viviani, 2016) separate labelling for these two vessel types. Future work could explore the use of these probabilistic digital atlases of the cerebral vasculature in patients with a vascular malformation. This approach could help to highlight the presence of anomalous vascular components, defined as vessels appearing in areas where there is a low probability of observing a healthy vessel.

No significant differences in the average hemispheric venous $\mathrm{SvO}_{2}$ were observed in or between any of the AVM patient groups or in the healthy volunteers. This result was expected in both healthy volunteers and GKR patients with successful AVM obliteration as their $\chi$ maps did not contain any anomalous venous patterns. The similar $\mathrm{SvO}_{2}$ in the two hemispheres of the pre-GKR patients could be linked to the inaccurate MVF segmentation of the AVM draining pattern discussed above. It could also be due to a low proportion of AVM venous voxels compared to the total number of segmented venous voxels in the brain hemisphere containing the AVM. Both these hypotheses would also explain why only one $\mathrm{SvO}_{2}$ peak was observed in the histogram analysis of the hemispheric $\mathrm{SvO}_{2}$ (see Inline Supplementary Fig. S4). Because the brain parenchyma is diamagnetic, partial volume effects between veins and the surrounding brain tissue could also contribute to decrease the $\mathrm{SvO}_{2}$ measured in the veins especially those with a diameter smaller than the acquired voxel size. The MVF algorithm mitigates partial volume effects by estimating the vessel diameters and using these to refine the vein segmentation. However, this approach does never fully remove partial voluming and it has been suggested (Bazin et al., 2016) that a future version of MVF could integrate the additional information on vein geometry and localization given by vascular trees or SWI. For the reduction of errors in $\chi$ quantification induced by partial voluming, other strategies have been proposed (Fan et al., 2014; Hsieh et al., 2015; McDaniel et al., 2017; Ward et al., 2017) and are discussed later in this section.

A negligible percentage $(<1 \%)$ of voxels with erroneous $\mathrm{SvO}_{2}$ values were excluded from the analysis of hemispheric $\mathrm{SvO}_{2}$. Such incorrect $\mathrm{SvO}_{2}$ values were linked to incorrect $\chi$ quantification in the corresponding voxels. In patients, this was possibly caused by the voxel's proximity to paramagnetic embolizing glue, hemosiderin deposits or non- $\chi$-induced dephasing due to flow. In both patients and healthy subjects, these errors possibly derived from the MVF method mislabeling image ridges caused by head motion or sensitivity encoding (SENSE)-induced artifacts as vessels.

The lack of $\chi$ contrast between ROIDv and surrounding brain tissue in the pre-GKR patients (Fig. 6b) compared with other veins (e.g., the SSS) supports the hypothesis that blood with a higher 
oxygenation than in healthy veins was collected by the AVMs' draining pattern due to the presence of arteriovenous shunting. This hypothesis was also confirmed by our finding that the $\mathrm{SvO}_{2}$ was always higher in $\mathrm{ROI}_{D V}$ than in $\mathrm{ROI}_{\text {sss }}$ (Fig. 7) and that the difference between $\mathrm{SvO}_{2}$ in $\mathrm{ROI}_{D V}$ and $\mathrm{ROI}_{S S S}$ was always higher than the difference between $\mathrm{SvO}_{2}$ in $\mathrm{ROI}_{\text {sss }}$ and a distinct healthy vein $\mathrm{ROI}$ (see Inline Supplementary Fig. S5). This is the first time that $\mathrm{SvO}_{2}$ has been non-invasively and directly measured in the veins draining an AVM.

Both the hemispheric $\mathrm{SvO}_{2}$ (see Inline Supplementary Fig. S3) and the $\mathrm{SvO}_{2}$ measured in $\mathrm{ROI}_{\text {sss }}$ (Fig.

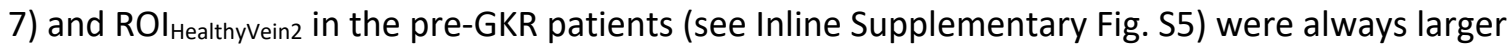
than $\mathrm{SvO}_{2}$ inferred from gold-standard PET measurements of oxygen extraction fraction (OEF), i.e., $61 \pm 6 \%$ (Hattori et al., 2004) or $57 \pm 6 \%$ (Bremmer et al., 2011). It must be noted that OEF is the percentage of oxygen extracted from arterial blood:

$$
\mathrm{OEF}=\mathrm{SaO}_{2}-\mathrm{SvO}_{2}
$$

where $\mathrm{SaO}_{2}$ is the arterial oxygen saturation and is assumed to be equal to $100 \% . \mathrm{SvO}_{2}$ in the healthy veins of the pre-GKR patients (Fig. 7 and Inline Supplementary Fig. S5) was also often larger than recent QSM-based measurements in the same vein (Fan et al., 2014), i.e., $63.8 \pm 3 \%$ across three healthy volunteers. Because of the inverse relationship between $\mathrm{SvO}_{2}$ and venous $\chi$ (Eq. 1 ), this overestimation of $\mathrm{SvO}_{2}$ was most likely linked to an underestimation of venous $\chi$. Several factors might have contributed to underestimating $\chi$. Firstly, the lack of flow compensation in the GRE acquisitions might have introduced errors in the GRE phase measured in the veins. In fact, Fan et al (Fan et al., 2014) used a fully velocity-compensated multi-echo GRE sequence for QSM whereas our QSM sequence was not flow-compensated. Secondly, QSM may be less accurate than PET because it involves the solution of an ill-posed inverse problem and the use of regularization in the local fieldto-susceptibility inversion method leads to underestimation in itself. We attempted to address this last issue by applying a correction for $\chi$ underestimation based on the point spread function of the modified dipole kernel (Schweser et al., 2013). However, in QSM the loss of information caused by the presence of zeros in the inverse magnetic dipole can never fully be recovered, and, even after correcting for $\chi$ underestimation induced by regularization, a slight overestimation of $\mathrm{SvO}_{2}$ compared to PET should always be expected. Thirdly, partial volume effects between veins and brain parenchyma at the boundaries of $\mathrm{ROI}_{\text {sss }}$ and $\mathrm{ROI}$ Dv might have inflated the $\mathrm{SvO}_{2}$ measurements. Several solutions have been proposed (Fan et al., 2014; Hsieh et al., 2015; McDaniel et al., 2017; Ward et al., 2017) to mitigate partial volume effects in venous ROIs. The two simplest approaches have involved either morphologically eroding the $\mathrm{ROI}$ or considering only the voxel with the maximum $\chi$ value within the ROI (Fan et al., 2014). Both these approaches discard a large number of voxels from the $\mathrm{ROI}$ and therefore make the resulting $\chi$ or $\mathrm{SvO}_{2}$ measurements highly sensitive to noise. Moreover, morphological erosion may leave few or no voxel in the ROI, especially in veins with a diameter of three voxels or fewer, which was the case of $\mathrm{ROI}_{\mathrm{DV}}$ and $\mathrm{ROI}_{\mathrm{sss}}$ in this study. Considering the maximum intensity voxel, instead, neglects information on potential regional $\chi$ variations due to gradients of oxygenation, whose exploration may be relevant in vascular disease. In alternative, other studies (Hsieh et al., 2015; McDaniel et al., 2017) have estimated and removed partial volume contributions from the affected voxels by modelling the complex GRE signal, whose phase component is sensitive to vein orientation. Such an approach, however, requires a vein to be parallel to the external magnetic field (McDaniel et al., 2017) or to not be tilted at the magic angle (Hsieh et al., 2015). To overcome limitations in vein orientation, another method (Ward et al., 2017) 
has calculated a parametric map of partial voluming using $\chi$ values measured by QSM. It must be noted that all these three methods (Hsieh et al., 2015; McDaniel et al., 2017; Ward et al., 2017) have assumed that veins can be modelled as long and relatively straight cylinders, but this assumption cannot always be fulfilled by AVM draining veins. Therefore, partial volume correction remains an open problem in the case of nonstandard vessel anatomies, such as those found in vascular malformations, and further work is needed in this direction. Finally, it must be remarked that, as shown by in silico experiments (Ward et al., 2017), perfect removal of partial volume effects does not overcome $\chi$ underestimation linked to the intrinsic limitations of the inverse problem solution in QSM. In this study, the potential influence of partial volume effects on ROI-based $\mathrm{SvO}_{2}$ measurements was mitigated by comparing the difference in $\mathrm{SvO}_{2}$ between healthy and AVM draining veins with the difference in $\mathrm{SvO}_{2}$ between two healthy veins. This experiment could not be carried out in an optimal way, because the heterogeneity of AVM anatomy made it impossible to consider the same pair of healthy veins in all pre-GKR patients. However, it provided evidence that differences in $\mathrm{SVO}_{2}$ between healthy and $\mathrm{AVM}$ draining veins are linked to disease rather than being purely caused by partial volume effects or technical limitations of QSM.

The benefit of using flow-compensated acquisition sequences for QSM of brain AVMs requires further investigation. Because of the tortuous nature of blood flow in and around the AVM, the assumptions underpinning conventional velocity compensation techniques (e.g., a plug flow model of venous blood flow) are probably violated in brain AVMs. One study (Ansari et al., 2013) using cardiac-gated and time-resolved 3D PCA has shown that both the feeding arteries and draining veins of a brain AVM are characterized by heterogeneous blood flow velocity distributions. In particular, venous blood flow velocities in an AVM's draining veins are substantially higher (i.e., higher than 30$50 \mathrm{~cm} / \mathrm{s}$ ) compared to normal venous velocities (i.e., $15-25 \mathrm{~cm} / \mathrm{s}$ ). Therefore, flow-induced $\chi$ shifts may be substantial in AVM vessels. To gain insight into the complexity of blood flow in and around AVMs and reduce sources of $\chi$ error due to turbulent flow, future work could involve building and imaging a blood flow phantom and analyzing previous results of studies of AVM hemodynamics (Ansari et al., 2013). This could enable improved flow compensation techniques for more accurate GRE imaging and QSM of brain AVMs.

A further limitation of this study was the fact that the pre-GKR and post-GKR patient groups did not include the same subjects. Future work could involve scanning the patients of the pre-GKR cohort at least three years after treatment to confirm obliteration of the AVM and evaluate the effects of GKR on hemispheric venous density and $\mathrm{SVO}_{2}$ at an individual level.

\section{Conclusion}

We have shown that QSM is useful in the characterization of vascular abnormalities associated with AVMs. $\chi$-based measures of hemispheric vessel density successfully differentiated brain hemispheres containing an AVM from the contralateral hemisphere. Therefore, similar measurements could be used as a tool for the $\chi$-based detection of brain AVMs.

Based on the intravascular $\chi$ values, higher $\mathrm{SvO}_{2}$ was measured in the AVM draining veins than in healthy veins, demonstrating that QSM can detect $\mathrm{SvO}_{2}$ alterations in brain AVMs. This is the first study to non-invasively and directly measure $\mathrm{SvO}_{2}$ in the veins draining an AVM and to confirm the physiological hypothesis that blood flowing through the arteriovenous shunt is more oxygenated than blood in healthy veins. 


\section{Acknowledgments}

Emma Biondetti was supported by the UK Engineering and Physical Research Council (EPSRC) (award number: 1489882); David L. Thomas was supported by the UCL Leonard Wolfson Experimental Neurology Centre (PR/ylr/18575).

The authors would like to thank Dr. Audrey Fan (Radiological Sciences Laboratory, Stanford University, Stanford, CA, USA) for useful discussions on methods for $\chi$-based vein segmentation and $\mathrm{SvO}_{2}$ measurement, and Dr. Pierre-Louis Bazin (Department of Neurology and Department of Neurophysics, Max Planck Institute for Human Cognitive and Brain Sciences, Leipzig, Germany; Social Brain Laboratory, Netherlands Institute for Neuroscience, Amsterdam, Netherlands; Spinoza Centre for Neuroimaging, Amsterdam, Netherlands) for his help with setting up the MVF algorithm for vein segmentation. The authors would also like to thank Drs. Marco Battiston, Rosa Cortese, Floriana De Angelis, Francesco Grussu and Marios Yiannakas (Queen Square MS Centre, UCL Institute of Neurology, University College London, London, UK) for their help with scanning the healthy volunteers.

\section{References}

Alsop, D.C., et al., 2015. Recommended implementation of arterial spin-labeled perfusion MRI for clinical applications: A consensus of the ISMRM perfusion study group and the European consortium for ASL in dementia. Magn Reson Med 73, 102-116, http://dx.doi.org/10.1002/mrm.25197.

Ansari, S.A., et al., 2013. Intracranial 4D flow MRI: toward individualized assessment of arteriovenous malformation hemodynamics and treatment-induced changes. AJNR Am J Neuroradiol 34, 1922-1928, http://dx.doi.org/10.3174/ajnr.A3537.

Bazin, P.-L., et al., 2016. Vessel Segmentation from Quantitative Susceptibility Maps for Local Oxygenation Venography. ISBI, pp. 1135-1138, http://dx.doi.org/10.1109/ISBI.2016.7493466.

Bednarz, G., et al., 2000. Combining stereotactic angiography and 3D time-of-flight magnetic resonance angiography in treatment planning for arteriovenous malformation radiosurgery. Int J Radiat Oncol Biol Phys 46, 1149-1154, https://www.ncbi.nlm.nih.gov/pubmed/10725625

Beriault, S., et al., 2015. Automatic SWI Venography Segmentation Using Conditional Random Fields. IEEE Trans Med Imaging 34, 2478-2491, http://dx.doi.org/10.1109/TMI.2015.2442236.

Bernier, M., et al., 2018. The morphology of the human cerebrovascular system. Hum Brain Mapp 39, 4962-4975, http://dx.doi.org/10.1002/hbm.24337.

Bremmer, J.P., et al., 2011. Day-to-day test-retest variability of CBF, CMRO2, and OEF measurements using dynamic 150 PET studies. Mol Imaging Biol 13, 759-768, http://dx.doi.org/10.1007/s11307010-0382-1.

Cetin, S., et al., 2016. Vessel Orientation Constrained Quantitative Susceptibility Mapping (QSM) Reconstruction. Springer International Publishing, Cham, http://dx.doi.org/10.1007/978-3-31946726-9 54.

Cronqvist, M., et al., 2006. Endovascular treatment of intracerebral arteriovenous malformations: procedural safety, complications, and results evaluated by MR imaging, including diffusion and 
perfusion imaging. AJNR Am J Neuroradiol 27, 162-176, https://www.ncbi.nlm.nih.gov/pubmed/16418378

Dammann, P., et al., 2013. The venous angioarchitecture of sporadic cerebral cavernous malformations: a susceptibility weighted imaging study at 7 T MRI. J Neurol Neurosurg Psychiatry 84, 194-200, http://dx.doi.org/10.1136/jnnp-2012-302599.

Dixon, E.C., 2018. Applications of MRI Magnetic Susceptibility Mapping in PET-MRI Brain Studies (PhD Thesis). Department of Medical Physics and Biomedical Engineering. University College London, London,

Dunas, T., et al., 2017. A Stereotactic Probabilistic Atlas for the Major Cerebral Arteries. Neuroinformatics 15, 101-110, http://dx.doi.org/10.1007/s12021-016-9320-y.

Durst, C.R., et al., 2016. Prevalence of dural venous sinus stenosis and hypoplasia in a generalized population. J Neurointerv Surg 8, 1173-1177, http://dx.doi.org/10.1136/neurintsurg-2015-012147.

Duyn, J.H., Schenck, J., 2017. Contributions to magnetic susceptibility of brain tissue. NMR Biomed 30, http://dx.doi.org/10.1002/nbm.3546.

Dymerska, B., et al., 2018. In vivo phase imaging of human epiphyseal cartilage at 7 T. Magn Reson Med 79, 2149-2155, http://dx.doi.org/10.1002/mrm.26858.

Essig, M., et al., 1999. High-resolution MR venography of cerebral arteriovenous malformations. Magn Reson Imaging 17, 1417-1425, http://dx.doi.org/10.1016/S0730-725X(99)00084-3.

Fan, A.P., et al., 2014. Quantitative oxygenation venography from MRI phase. Magn Reson Med 72, 149-159, http://dx.doi.org/10.1002/mrm.24918.

Fan, A.P., et al., 2016. Baseline oxygenation in the brain: Correlation between respiratory-calibration and susceptibility methods. Neuroimage 125, 920-931,

http://dx.doi.org/10.1016/i.neuroimage.2015.11.007.

Fonov, V., et al., 2011. Unbiased average age-appropriate atlases for pediatric studies. Neuroimage 54, 313-327, http://dx.doi.org/10.1016/i.neuroimage.2010.07.033.

Fonov, V.S., et al., 2009. Unbiased nonlinear average age-appropriate brain templates from birth to adulthood. Neuroimage 47, http://dx.doi.org/10.1016/s1053-8119(09)70884-5.

Friedlander, R.M., 2007. Clinical practice. Arteriovenous malformations of the brain. N Engl J Med 356, 2704-2712, http://dx.doi.org/10.1056/NEJMcp067192.

Fushimi, Y., et al., 2008. A developmental venous anomaly presenting atypical findings on susceptibility-weighted imaging. AJNR Am J Neuroradiol 29, E56, http://dx.doi.org/10.3174/ajnr.A1074.

Gasparotti, R., et al., 2011. New MR sequences in daily practice: susceptibility weighted imaging. A pictorial essay. Insights Imaging 2, 335-347, http://dx.doi.org/10.1007/s13244-011-0086-3.

Gelal, F., et al., 2014. Capillary telangiectasia of the brain: imaging with various MR techniques. Journal of the Belgian Society of Radiology 97, 233-238, http://dx.doi.org/10.5334/ibr-btr.90. 
George, U., et al., 2010. Susceptibility-weighted imaging in the evaluation of brain arteriovenous malformations. Neurol India 58, 608-614, http://dx.doi.org/10.4103/0028-3886.68668.

Gould, I.G., et al., 2017. The capillary bed offers the largest hemodynamic resistance to the cortical blood supply. J Cereb Blood Flow Metab 37, 52-68, http://dx.doi.org/10.1177/0271678X16671146.

Haacke, E.M., et al., 2004. Susceptibility weighted imaging (SWI). Magn Reson Med 52, 612-618, http://dx.doi.org/10.1002/mrm.20198.

Hansen, P.C., O'Leary, D.P., 1993. The Use of the L-Curve in the Regularization of Discrete III-Posed Problems. SIAM Journal on Scientific Computing 14, 1487-1503, http://dx.doi.org/10.1137/0914086.

Hattori, N., et al., 2004. Accuracy of a method using short inhalation of (15)O-O(2) for measuring cerebral oxygen extraction fraction with PET in healthy humans. Journal of nuclear medicine 45,765 770, http://www.ncbi.nlm.nih.gov/pubmed/15136624

Hodel, J., et al., 2013. Susceptibility-weighted angiography for the detection of high-flow intracranial vascular lesions: preliminary study. Eur Radiol 23, 1122-1130, http://dx.doi.org/10.1007/s00330$\underline{012-2690-0 .}$

Horsch, S., et al., 2014. Developmental venous anomaly in the newborn brain. Neuroradiology 56, 579-588, http://dx.doi.org/10.1007/s00234-014-1367-6.

Hsieh, C.Y., et al., 2015. An improved method for susceptibility and radius quantification of cylindrical objects from MRI. Magn Reson Imaging 33, 420-436, http://dx.doi.org/10.1016/i.mri.2015.01.004.

Huck, J., et al., 2018. High resolution atlasing of the venous brain vasculature from 7T quantitative susceptibility. bioRxiv [Preprint]. Cited on April 01, 2019, http://dx.doi.org/10.1101/444349.

Huisman, T.A., et al., 2010. Non-invasive imaging of intracranial pediatric vascular lesions. Childs Nerv Syst 26, 1275-1295, http://dx.doi.org/10.1007/s00381-010-1203-1.

Jagadeesan, B.D., et al., 2011. Postcontrast susceptibility-weighted imaging: a novel technique for the detection of arteriovenous shunting in vascular malformations of the brain. Stroke 42, 31273131, http://dx.doi.org/10.1161/STROKEAHA.111.623587.

Jain, V., et al., 2012. Investigating the magnetic susceptibility properties of fresh human blood for noninvasive oxygen saturation quantification. Magn Reson Med 68, 863-867, http://dx.doi.org/10.1002/mrm.23282.

Jenkinson, M., et al., 2012. Fsl. Neuroimage 62, 782-790, http://dx.doi.org/10.1016/j.neuroimage.2011.09.015.

Jenkinson, M., Smith, S., 2001. A global optimisation method for robust affine registration of brain images. Med Image Anal 5, 143-156, https://www.ncbi.nlm.nih.gov/pubmed/11516708

Kano, H., et al., 2012. Stereotactic radiosurgery for arteriovenous malformations, Part 3: outcome predictors and risks after repeat radiosurgery. J Neurosurg 116, 21-32,

http://dx.doi.org/10.3171/2011.9.JNS101741. 
Katayama, Y., et al., 1994. Continuous monitoring of jugular bulb oxygen saturation as a measure of the shunt flow of cerebral arteriovenous malformations. J Neurosurg 80, 826-833, http://dx.doi.org/10.3171/jns.1994.80.5.0826.

Knerlich-Lukoschus, F., et al., 2015. Cerebellar cavernous malformation in pediatric patients: defining clinical, neuroimaging, and therapeutic characteristics. J Neurosurg Pediatr 16, 256-266, http://dx.doi.org/10.3171/2015.1.PEDS14366.

Koltz, M.T., et al., 2013. Long-term outcome of Gamma Knife stereotactic radiosurgery for arteriovenous malformations graded by the Spetzler-Martin classification. J Neurosurg 118, 74-83, http://dx.doi.org/10.3171/2012.9.JNS112329.

Kressler, B., et al., 2010. Nonlinear regularization for per voxel estimation of magnetic susceptibility distributions from MRI field maps. IEEE Trans Med Imaging 29, 273-281, http://dx.doi.org/10.1109/TMI.2009.2023787.

Kukuk, G.M., et al., 2010. Cerebral arteriovenous malformations at 3.0 T: intraindividual comparative study of 4D-MRA in combination with selective arterial spin labeling and digital subtraction angiography. Invest Radiol 45, 126-132, http://dx.doi.org/10.1097/RLI.0b013e3181c7bcfe.

Le, T.T., et al., 2012. Identification of venous signal on arterial spin labeling improves diagnosis of dural arteriovenous fistulas and small arteriovenous malformations. AJNR Am J Neuroradiol 33, 6168, http://dx.doi.org/10.3174/ainr.A2761.

Lee, B.C., et al., 1999. MR high-resolution blood oxygenation level-dependent venography of occult (low-flow) vascular lesions. AJNR Am J Neuroradiol 20, 1239-1242, https://www.ncbi.nlm.nih.gov/pubmed/10472978

Lim, I.A., et al., 2013. Human brain atlas for automated region of interest selection in quantitative susceptibility mapping: application to determine iron content in deep gray matter structures. Neuroimage 82, 449-469, http://dx.doi.org/10.1016/j.neuroimage.2013.05.127.

Liu, C., et al., 2015. Susceptibility-weighted imaging and quantitative susceptibility mapping in the brain. J Magn Reson Imaging 42, 23-41, http://dx.doi.org/10.1002/jmri.24768.

Liu, T., et al., 2011. A novel background field removal method for MRI using projection onto dipole fields (PDF). NMR Biomed 24, 1129-1136, http://dx.doi.org/10.1002/nbm.1670.

Liu, T., et al., 2009. Calculation of susceptibility through multiple orientation sampling (COSMOS): a method for conditioning the inverse problem from measured magnetic field map to susceptibility source image in MRI. Magn Reson Med 61, 196-204, http://dx.doi.org/10.1002/mrm.21828.

Liu, T., et al., 2013. Nonlinear formulation of the magnetic field to source relationship for robust quantitative susceptibility mapping. Magn Reson Med 69, 467-476, http://dx.doi.org/10.1002/mrm.24272.

Lu, L., et al., 2011. Increased endothelial progenitor cells and vasculogenic factors in higher-staged arteriovenous malformations. Plast Reconstr Surg 128, 260e-269e, http://dx.doi.org/10.1097/PRS.0b013e3182268afd.

McDaniel, P., et al., 2017. Mitigation of partial volume effects in susceptibility-based oxygenation measurements by joint utilization of magnitude and phase (JUMP). Magn Reson Med 77, 1713-1727, http://dx.doi.org/10.1002/mrm.26227. 
Modat, M., et al., 2010. Fast free-form deformation using graphics processing units. Comput Methods Programs Biomed 98, 278-284, http://dx.doi.org/10.1016/j.cmpb.2009.09.002.

Morris, Z., et al., 2009. Incidental findings on brain magnetic resonance imaging: systematic review and meta-analysis. BMJ 339, b3016, http://dx.doi.org/10.1136/bmj.b3016.

Nabavizadeh, S.A., et al., 2014. Utility of susceptibility-weighted imaging and arterial spin perfusion imaging in pediatric brain arteriovenous shunting. Neuroradiology 56, 877-884, http://dx.doi.org/10.1007/s00234-014-1408-1.

Nissi, M.J., et al., 2015. Improved Visualization of Cartilage Canals Using Quantitative Susceptibility Mapping. PLoS One 10, e0132167, http://dx.doi.org/10.1371/journal.pone.0132167.

Ourselin, S., et al., 2001. Reconstructing a 3D structure from serial histological sections. Image and Vision Computing 19, 25-31, http://dx.doi.org/10.1016/s0262-8856(00)00052-4.

Ozbay, P.S., et al., 2015. Effect of respiratory hyperoxic challenge on magnetic susceptibility in human brain assessed by quantitative susceptibility mapping (QSM). NMR Biomed 28, 1688-1696, http://dx.doi.org/10.1002/nbm.3433.

Passat, N., et al., 2005. Region-growing segmentation of brain vessels: an atlas-based automatic approach. J Magn Reson Imaging 21, 715-725, http://dx.doi.org/10.1002/jmri.20307.

Pollock, J.M., et al., 2011. Response of arteriovenous malformations to gamma knife therapy evaluated with pulsed arterial spin-labeling MRI perfusion. AJR Am J Roentgenol 196, 15-22, http://dx.doi.org/10.2214/AJR.10.5290.

Reichenbach, J., et al., 1998. High-resolution venography of the brain using magnetic resonance imaging. Magnetic Resonance Materials in Biology, Physics, and Medicine 6, 62-69, http://dx.doi.org/10.1016/s1352-8661(98)00011-8.

Reichenbach, J.R., Haacke, E.M., 2001. High-resolution BOLD venographic imaging: a window into brain function. NMR Biomed 14, 453-467, https://www.ncbi.nlm.nih.gov/pubmed/11746938

Reichenbach, J.R., et al., 2001. High-resolution blood oxygen-level dependent MR venography (HRBV): a new technique. Neuroradiology 43, 364-369,

https://www.ncbi.nlm.nih.gov/pubmed/11396739

Rojas-Villabona, A., et al., 2017. Triple Magnetic Resonance Angiography (triple-MRA) for Planning of Gamma Knife Radiosurgery of Brain Arteriovenous Malformations. International Society for Magnetic Resonance in Medicine,

Schenck, J.F., 1996. The role of magnetic susceptibility in magnetic resonance imaging: MRI magnetic compatibility of the first and second kinds. Med Phys 23, 815-850,

http://dx.doi.org/10.1118/1.597854.

Schweser, F., et al., 2013. Toward online reconstruction of quantitative susceptibility maps: superfast dipole inversion. Magn Reson Med 69, 1582-1594, http://dx.doi.org/10.1002/mrm.24405.

Serres, B., et al., 2015. Automatic Segmentation of the Venous Vessel Network Based on Quantitative Susceptibility Maps and its Applications. International Society for Magnetic Resonance in Medicine, p. 169, 
Shmueli, K., et al., 2009. Magnetic susceptibility mapping of brain tissue in vivo using MRI phase data. Magn Reson Med 62, 1510-1522, http://dx.doi.org/10.1002/mrm.22135.

Spees, W.M., et al., 2001. Water proton MR properties of human blood at 1.5 Tesla: magnetic susceptibility, $T(1), T(2), T^{*}(2)$, and non-Lorentzian signal behavior. Magn Reson Med 45, 533-542, http://dx.doi.org/10.1002/mrm.1072.

Starke, R.M., et al., 2017. Stereotactic radiosurgery for cerebral arteriovenous malformations: evaluation of long-term outcomes in a multicenter cohort. J Neurosurg 126, 36-44, http://dx.doi.org/10.3171/2015.9.JNS151311.

Straub, S., et al., 2017. Suitable reference tissues for quantitative susceptibility mapping of the brain. Magn Reson Med 78, 204-214, http://dx.doi.org/10.1002/mrm.26369.

Thomas, B., et al., 2008. Clinical applications of susceptibility weighted MR imaging of the brain - a pictorial review. Neuroradiology 50, 105-116, http://dx.doi.org/10.1007/s00234-007-0316-z.

Tong, K.A., et al., 2008. Susceptibility-weighted MR imaging: a review of clinical applications in children. AJNR Am J Neuroradiol 29, 9-17, http://dx.doi.org/10.3174/ajnr.A0786.

Viviani, R., 2016. A Digital Atlas of Middle to Large Brain Vessels and Their Relation to Cortical and Subcortical Structures. Front Neuroanat 10, 12, http://dx.doi.org/10.3389/fnana.2016.00012.

Vollherbst, D.F., et al., 2018. Imaging artifacts of Onyx and PHIL on conventional CT, cone-beam CT and MRI in an animal model. Interv Neuroradiol 24, 693-701, http://dx.doi.org/10.1177/1591019918782692.

Wang, Y., Liu, T., 2015. Quantitative susceptibility mapping (QSM): Decoding MRI data for a tissue magnetic biomarker. Magn Reson Med 73, 82-101, http://dx.doi.org/10.1002/mrm.25358.

Ward, P.G., et al., 2017. Improved Quantification of Cerebral Vein Oxygenation Using Partial Volume Correction. Front Neurosci 11, 89, http://dx.doi.org/10.3389/fnins.2017.00089.

Ward, P.G.D., et al., 2018. Combining images and anatomical knowledge to improve automated vein segmentation in MRI. Neuroimage 165, 294-305, http://dx.doi.org/10.1016/j.neuroimage.2017.10.049.

Weisskoff, R.M., Kiihne, S., 1992. MRI susceptometry: image-based measurement of absolute susceptibility of MR contrast agents and human blood. Magn Reson Med 24, 375-383, https://www.ncbi.nlm.nih.gov/pubmed/1569876

Wharton, S., Bowtell, R., 2010. Whole-brain susceptibility mapping at high field: a comparison of multiple- and single-orientation methods. Neuroimage 53, 515-525, http://dx.doi.org/10.1016/j.neuroimage.2010.06.070.

Wolf, R.L., et al., 2008. Arteriovenous shunt visualization in arteriovenous malformations with arterial spin-labeling MR imaging. AJNR Am J Neuroradiol 29, 681-687, http://dx.doi.org/10.3174/ajnr.A0901.

Xu, B., et al., 2014. Flow compensated quantitative susceptibility mapping for venous oxygenation imaging. Magn Reson Med 72, 438-445, http://dx.doi.org/10.1002/mrm.24937. 
Yushkevich, P.A., et al., 2006. User-guided 3D active contour segmentation of anatomical structures: significantly improved efficiency and reliability. Neuroimage 31, 1116-1128,

http://dx.doi.org/10.1016/j.neuroimage.2006.01.015. 